\title{
Chinese Guideline on allergen immunotherapy for allergic rhinitis
}

\author{
Yixiao Bao ${ }^{1 *}$, Jianjun Chen ${ }^{2 *}$, Lei Cheng ${ }^{3,4 *}$, Yinshi Guo ${ }^{5 *}$, Suling Hong ${ }^{6 *}$, Weijia Kong ${ }^{2 *}$ He Lai $^{7^{*}}$, \\ Houyong $\mathrm{Li}^{8 *}$, Huabin $\mathrm{Li}^{8 *}$, Jing $\mathrm{Li}^{9 *}$, Tianying $\mathrm{Li}^{1{ }^{1 *}}$, Xiaoping $\mathrm{Lin}^{1{ }^{1 *}}$, Shixi $\mathrm{Liu}^{12 *}$, Zheng Liu ${ }^{13 *}$, Hongfei \\ Lou $^{14,15 *}$, Juan Meng ${ }^{12 *}$, Qianhui Qiu ${ }^{16 *}$, Kunling Shen ${ }^{17 *}$, Wei Tang ${ }^{18 *}$, Zezhang Tao ${ }^{19 *}$, Chengshuo

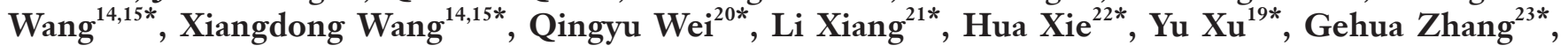 \\ Yuan Zhang ${ }^{14,15 *}$, Yiwu Zheng ${ }^{2 *}$, Yuxiang $\mathrm{Zhi}^{25 *}$, Dehua Chen ${ }^{10}$, Haiyu Hong ${ }^{10}$, Quansheng $\mathrm{Li}^{20}$, Lin $\mathrm{Liu}^{3}$, \\ Yifan Meng ${ }^{14,15}$, Nan Wang ${ }^{13}$, Yihui Wang ${ }^{1}$, Yue Zhou ${ }^{2}$, Luo Zhang ${ }^{14,15,26}$; Chinese Society of Allergy (CSA) \\ and Chinese Allergic Rhinitis Collaborative Research Group (C2AR2G)
}

\begin{abstract}
${ }^{1}$ Department of Pediatric Respiratory Medicine, Pubin Children Hospital, Shanghai Children Medical Center Affiliated to Shanghai Jiao Tong University School of Medicine, Shanghai 200092, China; ${ }^{2}$ Department of Otorhinolaryngology, Union Hospital, Tongji Medical College, Huazhong University of Science and Technology, Wuhan 430022, China; ${ }^{3}$ Department of Otorhinolaryngology, the First Affiliated Hospital, Nanjing Medical University, Nanjing 210029, China; ${ }^{4}$ International Centre for Allergy Research, Nanjing Medical University, Nanjing 210029 , China; ${ }^{5}$ Department of Allergy \& Immunology, Renji Hospital, School of Medicine, Shanghai Jiao Tong University, Shanghai 200127, China; ${ }^{6}$ Department of Otorhinolaryngology, the First Affiliated Hospital of Chongqing Medical University, Chongqing 400016, China; ${ }^{7}$ Department of Allergy, the Second Affiliated Hospital of Guangzhou Medical University, Guangzhou 510260, China; ${ }^{8}$ Department of Otolaryngology, Head and Neck Surgery, Affiliated Eye, Ear, Nose and Throat Hospital, Fudan University, Shanghai 200031, China; ${ }^{9}$ State Key Laboratory of Respiratory Disease, Allergy and Clinical Immunology, the First Affiliated Hospital of Guangzhou Medical University, Guangzhou 510120, China; ${ }^{10}$ Department of otolaryngology, the First Affiliated Hospital of Sun Yat-sen University, Guangzhou 510080, China; ${ }^{11}$ The PLA Center of respiratory and allergic disease diagnosing and management, Shenyang 110016, China; ${ }^{12}$ Department of Otolaryngology, West China Hospital, Sichuan University, Chengdu 610041, China; ${ }^{13}$ Department of Otolaryngology Head and Neck Surgery, Tongji Hospital, Tongji Medical College, Huazhong University of Science and Technology, Wuhan 430030, China; ${ }^{14}$ Department of Otolaryngology Head and Neck Surgery, Beijing TongRen Hospital, Capital Medical University, Beijing 100730, China; ${ }^{15}$ Beijing Key Laboratory of Nasal Diseases, Beijing Institute of Otolaryngology, Beijing 100005, China; ${ }^{16}$ Department of Otorhinolaryngology, Zhujiang Hospital, Southern Medical University, Guangzhou 510282, China; ${ }^{17}$ Department of Pediatric Respiratory Medicine, Beijing Children's Hospital, Capital Medical University, National Center for Children's Health, Beijing 100045, China; ${ }^{18}$ Department of Pulmonary and Critical Care Medicine, Shanghai Ruijin Hospital affiliated to Shanghai Jiaotong University, School of Medicine, Shanghai 200025, China; ${ }^{19}$ Department of Otolaryngology, Renmin Hospital of Wuhan University, Wuhan 430060, China; ${ }^{20}$ Department of Allergy, NO.202 Hospital of PLA, Shenyang 110003, China; ${ }^{21}$ Department of Allergy, Beijing Children's Hospital, Capital Medical University, National Center for Children's Health, Beijing 100045, China; ${ }^{22}$ Department of Respiratory Medicine, the General Hospital of Shenyang Military Region, Shenyang 110016, China; ${ }^{23}$ Department of Otolaryngology Head and Neck Surgery, The Third Affiliated Hospital of Sun Yat-sen University, Guangzhou 510630, China; ${ }^{24}$ Scientific Affairs, ALK, Guangzhou 510300, China; ${ }^{25}$ Department of Allergy, Peking Union Medical College Hospital, Chinese Academy of Medical Sciences \& Peking Union Medical College, Beijing 100720, China; ${ }^{26}$ Department of Allergy, Beijing TongRen Hospital, Capital Medical University, Beijing 100730, China

*These authors contributed equally to this work.

Correspondence to: Luo Zhang, MD. Beijing Institute of Otolaryngology, No. 17, HouGouHuTong, DongCheng District, Beijing 100005, China. Email: dr.luozhang@139.com.
\end{abstract}

\begin{abstract}
The present document is based on a consensus reached by a panel of experts from Chinese Society of Allergy (CSA) and Chinese Allergic Rhinitis Collaborative Research Group (C2AR2G). Allergen immunotherapy (AIT), has increasingly been used as a treatment for allergic rhinitis (AR) globally, as it has been shown to provide a long-term effect in improving nasal and ocular symptoms, reducing medication need, and improving quality of life. AIT is currently the only curative intervention that can potentially modify the immune system in individuals suffering from AR and prevent the development of new sensitization and the progression of disease from AR to asthma. Although the use of AIT is becoming more acceptable in China, to date no AR immunotherapy guideline from China is available for use by the international community. This document has thus been produced and covers the main aspects of AIT
\end{abstract}


undertaken in China; including selection of patients for AIT, the allergen extracts available on the Chinese market, schedules and doses of allergen employed in different routes of AIT, assessment of effect and safety, patients' administration and follow-up, and management of adverse reactions. The Chinese guideline for AR immunotherapy will thus serve as a reference point by doctors, healthcare professionals and organizations involved in the AIT of AR in China. Moreover, this guideline will serve as a source of information for the international community on AIT treatment strategies employed in China.

Keywords: Allergen immunotherapy (AIT); allergic rhinitis (AR); guideline; China

Submitted Oct 13, 2017. Accepted for publication Oct 20, 2017.

doi: $10.21037 /$ jtd.2017.10.112

View this article at: http://dx.doi.org/10.21037/jtd.2017.10.112

\section{Introduction}

Allergic rhinitis (AR) is an inflammatory disease of the nasal mucosa, induced by an immunoglobulin E ( $\mathrm{IgE}$ )-mediated reaction in allergen-sensitized subjects. It is characterized by sneezing, rhinorrhea, nasal congestion and nasal pruritus, which are often accompanied by ocular pruritus, redness and/or lacrimation (1). In view of the large numbers of patients with comorbid rhinitis and asthma, it has been suggested that AR and asthma may be regarded as "one airway, one disease" (2). Moreover, it is generally thought that there exists an "allergic march" (3), the natural history of atopic manifestations characterized by a typical sequence of $\mathrm{IgE}$ antibody responses and clinical symptoms which may appear early in life, which persists over years or decades and often remits spontaneously with age. However, although not life threatening, the symptoms of AR as well as the comorbid diseases are frequently bothersome, adversely affect work, psychological health (4-7) and quality of life, as well as imposing a significant socioeconomic burden on both the individual and society (8-11).

Epidemiological studies have revealed that the prevalence of AR has increased progressively in the moredeveloped countries, and currently affects up to $40 \%$ of the population worldwide (12-15). Likewise, rising trend of AR has also been observed globally in the past decades; with a widely varying prevalence particularly in the developing nations, including in China (16). Indeed, one nationwide population-based study assessed self-reported AR using validated questionnaire-based telephone interviews in over 38,000 adult subjects in 11 major cities across China, from September 2004 to May 2005, and demonstrated that while the prevalence of AR was $11.1 \%$ among the entire study cohort, there was highly variability in prevalence of AR among the different cities; ranging from $8.7 \%$ in Beijing to $24.1 \%$ in Urumqi (17). Similarly, a survey of a total of 23,791 children aged 6-13 years in 8 metropolitan capital cities of provinces in four regions across China, during November and December 2005, demonstrated that the mean prevalence of childhood AR was $9.8 \%$, and ranged from $3.9 \%$ in Xi'an to $16.8 \%$ in Guangzhou (18). A more recent study has further reported a progressive overall increase in the prevalence of self-reported AR during a 6-year period in the general Chinese adult population in major cities (19). Compared with AR prevalence of $11.1 \%$ in the survey in 2005 , the standardized prevalence of adult AR in 18 major cities was $17.6 \%$ in 2011; with the highest AR prevalence of $23 \%$ reported for Shanghai and lowest AR prevalence of $9.8 \%$ reported for Chengdu. These findings clearly suggest that increasing trend and the instability of the prevalence of $\mathrm{AR}$ in China, which may not have peaked yet.

The management of AR includes allergen avoidance, pharmacotherapy, allergen immunotherapy (AIT), and patient education (1). Thus, identifying the major local allergens is the first step to AR management involving diagnosis, prevention and AIT. Identification of the local prevailing allergens is particularly important as the type of allergen inducing AR varies significantly among regions due to the effect of geographic, climatic, and humanistic factors. Zhang et al. (16) summarized the patterns of sensitization to inhalant allergens among AR patients in mainland China and demonstrated that the prevalence and type of aeroallergens were different in various cities and regions. Dust mites were reported as the most common allergen in many regions, whereas Goosefoot and Lupulus (instead of dust mites) were the main aeroallergens in north-western China. Compared with adults, studies of the sensitizing inhaled allergens in children with AR suggested that a 
wider spectrum and Alternaria tenuis as well as mixed fungi were more common in children than adults. In $2009, \mathrm{Li}$ and colleagues (20) surveyed 6,304 patients suffering from asthma and/or rhinitis in 17 cities from four regions of China and showed that Dermatophagoides farina (Der $f$ ) and Dermatophagoides pteronyssinus (Der $p$ ) were the predominant aeroallergens in perennial/persistent AR individuals in China.

As a hallmark of AR treatment, AIT is currently the only curative intervention that can potentially modify the immune system of individuals suffering from $A R$, and thus affect the natural course of allergic disease (21). Research on and the clinical practice of AIT has shown remarkable progress since Noon (22) first described immunotherapy for AR in 1911. The long-term effect, efficacy and safety of AIT have been investigated and confirmed in many clinical trials and studies (23-25). However, AIT has not become a common and widely accepted treatment option for AR in China, and it is available in only a few major cities. The limited use of AIT in China may be attributable to several factors; including insufficient acceptance of AIT by doctors and patients, the potential risk of anaphylaxis, and the inconvenience of allergen administration together with the relatively high cost of treatment. With regard to the inconvenience of AIT, it is necessary to design administration schedules that shorten the build-up phase without increasing the rate of adverse reactions. Clusteror rush-specific subcutaneous immunotherapy (SCIT) schedules have been proven to be good alternatives in terms of their safety as well efficiency. It is worth mentioning that the cluster schedule reported by Zhang and colleagues (26) in 2009 as a safe alternative to the conventional schedule with the advantage of achieving clinical effectiveness sooner has subsequently been referenced in several international guidelines of immunotherapy for allergy $(27,28)$. More recently, a prospective, open-IV clinical trial by Qiu and colleagues (29) has confirmed the safety and efficacy of rush immunotherapy (RIT) and conventional immunotherapy in Chinese AR patients. Zhou and colleagues (30) investigated the current trends in the awareness and application status of AIT among Chinese ENT (ear, nose, and throat) specialists across China and concluded that whilst most of the specialists appeared to be in agreement with recent AIT progress and international guidelines, many areas still need to enhance the standardization and use of AIT in China. In order to promote research and clinical practice on $\mathrm{AR}$ immunotherapy at all levels in China, the Chinese Allergic Rhinitis Collaborative Research Group (C2AR2G) reviewed the evidence on allergen specific SCIT in AR available in the literature (31). Furthermore, the Chinese Society of Allergy (CSA) organized a panel of experts to reach a more comprehensive consensus concerning not only SCIT but also SLIT in China, and to produce a guideline document in English. Since the recommendations in the guideline have been proposed by some specialists, the document still needs to be validated and commented by other experts from China, as well as the world. The validated Chinese guideline for AR immunotherapy will serve as the reference point by doctors, healthcare professionals and organizations involved in the AIT of AR in China and help to facilitate the development of relevant local standards of care and protocols in the management of AR patients. Moreover, this guideline will serve as a source of information for the international community on AIT treatment strategies employed in China.

\section{Significance of AIT}

AIT is an important treatment strategy for patients with AR aimed at rebalancing the response to allergens. AIT involves the administration of gradually increasing doses of an allergen extract, to which an individual is allergic, such that the incremental increases in the allergen lead to desensitized or hypo-sensitized of the immune system towards subsequent natural exposure to that allergen; thus, resulting in long-term relief of the symptoms of allergy and improvement of quality of life of the individual.

\section{Modifying effect of AIT on allergic disease}

\section{Adults}

AIT was empirically developed in 1911 to treat AR before the discovery of IgE (32). Noon (22) first speculated that pollens contained "toxins" and injecting hay fever patients with small amounts of this pollen "toxin" would help to achieve a state of immunity, like a vaccine. Subcutaneous injection of allergen extract, also known as allergy shots, has since been the historical and predominant method of administration. Indeed, a nationwide cross-sectional survey assessing the trends in specific immunotherapy for AR in China has recently shown that conventional subcutaneous injection was the treatment option, which was most highly recommended by $96.2 \%$ of the Chinese ENT Specialists surveyed (30). However, sublingual immunotherapy (SLIT) has rapidly been established with high scientific credibility and become the most viable alternative to SCIT. As 
house dust mites (HDM) have been shown to be the most prevalent allergens in patients with $\mathrm{AR}$ and atopic asthma in multi-center study of prevalence of sensitization in patients with AR and atopic asthma in Mainland China (20), the Chinese guidelines recommend that AR patients who are allergic to one or two aeroallergens and particularly sensitized to HDM, should be treated by AIT (30).

Several studies have demonstrated that both SCIT and SLIT can significantly improve the nasal symptoms, reduce the need for symptomatic medication, and improve the quality of life during and after therapy, in adult AR patients $(1,33)$. Compared to the pharmacological therapies, the inherent benefit of AIT is the potential to treat all clinical expressions of respiratory allergic disease (34). Furthermore, in the long-term AIT can produce economic savings due to a combination of reduced drug usage and improvement of quality of life (35).

The association between AR and asthma has been firmly established. AR and asthma share multiple common pathophysiological processes, and as AR frequently precedes the onset of asthma, this opens a window of opportunity for early intervention and possibly even prevention of asthma (36). A study investigating the effects of long-term therapeutic and preventive effects of AIT in AR patients with/without asthma allergic to HDM has demonstrated that a 3-year course of SCIT obtained a long-term effect of improving clinical symptoms and reducing the risk of development of asthma (37). Similarly, other studies have demonstrated that AIT can also reduce the development of new allergic sensitizations in AR patients (38-40). A recent meta-analysis has assessed randomized controlled studies investigating the preventive effects of AIT in the development of first allergic disease in healthy asymptomatic individuals and concluded that although AIT reduced the short-term risk of patients with AR developing asthma, there was no consistent evidence from the studies that AIT reduced the short-term risk of the onset of a first allergic disease in adults (41). However, the effect of AIT in reducing the risk of developing the first allergic disease over the longer term was not investigated and, thus this issue remains an open question, which needs to be addressed in future studies.

\section{Children}

AIT can prolong symptoms remission after discontinuation of treatment in children with AR (42-48). Significantly lower symptom scores and better quality of life scores were found in children treated with SCIT for 3 years, compared with either baseline scores or in children treated with conventional medication (42). Furthermore, the benefits lasted for up to the 5 th year after the end of SCIT. Subjects with mono-/poly-sensitization obtained the same benefits from SCIT. Similarly, significant improvements in symptoms and medication scores have been demonstrated in HDM-sensitized children with AR treated with a combination of SLIT for 2 years and symptomatic medication, compared with children treated with medication only (44). Moreover, 2 years after withdrawal of therapy, the symptom scores, medication score and subjective satisfaction in 11 years old children treated with SLIT were better than those in 4-5 years old group. Chen and colleagues (43) investigated the onset time and efficacy of SLIT in 140 children (aged 4-14 years) with perennial moderate to severe HDM-induced AR. The authors showed that total nasal symptom score (TNSS), total medication score (TMS) and visual analogue scale (VAS) of both younger children group (aged 4-8 years) and older children group (aged 9-14 years) decreased significantly after 3, 6, 12 and 24 months of treatment. Indeed, Weng and colleagues (49) found the effect of SLIT in children was better compared to that in adults. Jacobsen and colleagues (24) administered SCIT for 3 years to patients with $\mathrm{AR}$ and conjunctivitis, and followed them for up to 10 years after initiation of SCIT. The authors demonstrated that SCIT not only resulted in significant improvements in symptoms at the 10 -year follow-up compared to non-SLIT, but also showed a significant longitudinal protective effect against development of asthma and bronchial hyper-responsiveness (24).

Collectively these studies indicate that AIT has important modifying effects on allergic diseases; helping to achieve better control of disease, reduce medication dosage, and prevent development of new sensitization and subsequent asthma.

\section{Prevention of new sensitization}

\section{The effect of AIT in preventing new sensitizations}

Several studies have evaluated the effect of AIT in preventing new sensitisations in Chinese asthmatic and AR patients. One study by Song and colleagues (50) investigated efficacy of SIT with standardized allergen vaccine or no SCIT with only symptomatic therapy for 3 years in asthmatic children with mite allergy; by assessing skin prick test (SPT), pulmonary function, serum specificity $\operatorname{IgE}$ ( $\operatorname{sgE}$ ) and new sensitizations. The authors demonstrated that children receiving SCIT showed significantly improved 
clinical symptom scores, lung function, and skin test results compared to children treated with symptomatic therapy. Furthermore, none of the children receiving SCIT demonstrated new sensitizations, whereas $47.5 \%$ of children receiving symptomatic therapy demonstrated new sensitizations to pollens (wormwood, ragweed), animal dander (dog and cat), fungi and cockroach (50).

Another study by Song and colleagues (51) assessed the efficacy of SCIT in patients with AR, and demonstrated that the blood eosinophil numbers, skin test index, rhinitis symptom scores and drug scores were all decreased significantly after SCIT for 3 years, compared to symptomatic therapy. Moreover, no patient with AR developed asthma after SCIT treatment group, and only $2.1 \%$ of patients developed new allergen sensitizations. In contrast, patients $17.4 \%$ of patients treated with symptomatic therapy developed asthma and 32.6\% patients demonstrated new sensitizations, to wormwood, ragweed and tree pollens, animal dander and cockroach. It was suggested that the earlier AIT is used, the greater is its preventive effect on the development of new sensitization in AR.

A more recent study by Song and colleagues (52) has evaluated the long-term efficacy of HDM-SCIT in patients with AR. The authors demonstrated that clinical symptom scores, drug scores and skin test result were all improved significantly after SCIT for 3 years, compared to before SCIT and compared to symptomatic therapy for 3 years. Moreover, the curative effect of SCIT was maintained for another 2 years after termination of SCIT and no patient in the SCIT group developed asthma, while only $4.7 \%$ of patients developed new sensitizations; as indicated by both SPT and sIgE positive tests for wormwood and dog dander in two patients. In contrast $22.0 \%$ of patients in symptomatic therapy developed asthma and $41.5 \%$ of patients were found to be newly sensitized to wormwood (6 cases), ragweed (5 cases), dog hair (3 cases), tree pollen ( 2 cases) and cockroach (1 case).

Marogna and colleagues (53) have assessed the efficacy of SLIT for 3 years in children with AR with/without intermittent asthma, and demonstrated that SLIT was also more effective than conventional drug therapy in reducing the onset of new sensitizations; as indicated by development of new sensitizations in $3.1 \%$ of SLIT patients and $34.8 \%$ of conventional drug therapy patients (odds ratio, 16.85; $95 \%$ confidence interval, 5.73-49.13). Moreover, SLIT also reduced the development of mild persistent asthma and reduced bronchial hyperreactivity in children with respiratory allergy to a significantly greater level than conventional drug therapy.

The findings of Marogna and colleagues (53) have been confirmed by a recent study which investigated the effects of SLIT on new sensitization in Chinese AR children aged 3-13 years (54). Assessment of the number of positive SPTs before and after treatment demonstrated that the onset of new sensitization was observed in only $3.55 \%$ of children treated by SLIT for 12 months compared with a significantly greater number of $27.27 \%$ of children treated by standard drug therapy. Moreover, the number of positive allergens decreased in $11.35 \%$ of children in only the SLIT group, and asthma medication use was significantly decreased by the end of the study.

\section{The risk of development of new sensitization}

Patients with new sensitization have been shown to have higher atopy scores and medication scores for both rhinitis and asthma than patients without new sensitization, after 5 years of AIT treatment $(50-52,55,56)$. Interestingly, one study has demonstrated that the risk of development of new sensitization was 3 -fold higher in the control group than in the SIT-aq (SIT with aqueous extracts) group and 4-fold higher in the control group than in the SIT-ad (SIT with adsorbed extracts) group (57). Some longitudinal studies (57-59) have reported an increase in the sensitization rates from childhood to adulthood. While one study in children concluded that the evolution from mono- to polysensitization was age-related (58), another study has indicated that the rate of development of polysensitization is dramatically increased in previously mono-sensitized children after 2 to 10 years from the first diagnosis (59).

There is some evidence that patients suffering from comorbid asthma and rhinitis are significantly more likely to develop new sensitizations, compared to patients with only rhinitis (39). Furthermore, it has been suggested that genetic predisposition of an individual towards developing a type 2 helper $\mathrm{T}$ cell (Th2) response to specific allergens is a key determinant in the development of new sensitization (39).

\section{Cost effectiveness of immunotherapy}

Immunotherapy is the only treatment to date, which can modify the natural course of AR, however, it is costly due to a variety of factors such as use of high purity allergen extracts, administration by trained health-care specialists, etc., and needs long term complete treatment for a 
satisfactory outcome. Cost effectiveness analysis is thus important in health-care decision-making relevant to the patients, physicians, and the companies, which manufacture and supply the immunotherapy products (60).

Several individual studies and systematic reviews have been published, which suggests that immunotherapy is cost-effective as compared with standard symptomatic pharmacotherapy for AR and AR accompanied with asthma (ARAS) (61-67). These studies have consistently concluded that immunotherapy for 3 years was cost effective compared to pharmacotherapy with regard to symptom scores and quality of life improvements. Indeed, evidence indicates that immunotherapy is more cost-effective than standard symptomatic treatment when the social perspective and costs of productivity loss are taken into account (61-67). Furthermore, the cost effectiveness of immunotherapy is likely to be of greater magnitude when the long-term efficacy after termination of AIT treatment and the preventive effects of AIT on development of asthma and new sensitizations to allergens are considered (68-71).

Most studies investigating the cost-effectiveness of immunotherapy for AR have been conducted in Europe and in the United States, and thus the findings of these studies need to be interpreted with caution when generalizing their applicability to China and other countries from outside these regions. This is particularly the case because differences exist between countries; for example, different patient populations, availability and use of different immunotherapy products, different regulations and real-life practices, different medicinal and healthcare costs, etc.

Presently, three SCIT vaccines are available for use in China. The vaccine produced by one domestic company (Xinhualian company, Beijing, China) is used in about 30 hospitals, and costs about 2,780 RMB (400 US dollars) per year during the treatment of one patient. The other two products are available from Merck in Germany and ALK in Denmark, and both products cost about 800 US dollars per year during the treatment of one patient. Similarly, only one SLIT vaccine is currently available and used in China. This vaccine is produced by a domestic company (Wolwo company, Shanghai, China), and costs about 600 US dollars per year during the treatment of one patient.

There is presently a marked paucity of data on cost effectiveness of AIT for AR or ARAS in China. One study by Chen and colleagues (72) has recently compared the cost effectiveness of SCIT versus standard pharmacotherapy for HDM-sensitized ARAS patients, in real-life practice for the first year. The authors found that although cost of SCIT for 1 year was higher than that for standard pharmacotherapy, the cost effectiveness ratio of SCIT was significantly better than that of standard pharmacotherapy for nasal symptom scores (251.7 vs. 517.4 dollars) and asthma symptom scores (701.3 vs. 862.4 dollars) (72).

In view of these studies, it is clear that more welldesigned cost effectiveness studies of AIT investigating larger numbers of patients treated and observed over longer periods are required in Chinese AR and ARAS patients in order that better strategies can be developed for recommendation and wider use of AIT in China.

\section{Algorithm and annotations for immunotherapy}

In principle, AIT can be of benefit to all patients with typical history and symptoms of AR together with positive skin test and/or detectable serum sIgE. In clinical practice, however, AIT should be considered particularly in patients with moderate/severe intermittent or persistent AR, who do not respond sufficiently to current pharmacotherapy $(1,73,74)$ (Figure 1).

\section{Routes of administration}

Various routes of administration have been utilized clinically for administration of allergen in AIT, however, subcutaneous and sublingual routes of allergen administration are most commonly employed.

\section{Subcutaneous administration of allergen}

Subcutaneous administration of allergen is the most universal route of AIT for allergic diseases. In 1911, Noon and Freeman first injected grass pollen extracts subcutaneously to treat patients suffering from hay fever (22). Over a century to date, this route is still used to treat allergic asthma, allergic rhinoconjunctivitis and hymenoptera hypersensitivity (75). This is particularly because the efficacy and safety of SCIT has been widely confirmed in clinical trials, although the main disadvantage of the technique remains the inconvenience of repeated injection in a clinical setting and the presence of some systemic side effects depending on the extract used.

\section{Sublingual administration of allergen}

Sublingual administration of allergen has become as an effective alternative to subcutaneous administration of allergen for AIT in recent decades, in spite of SCIT 


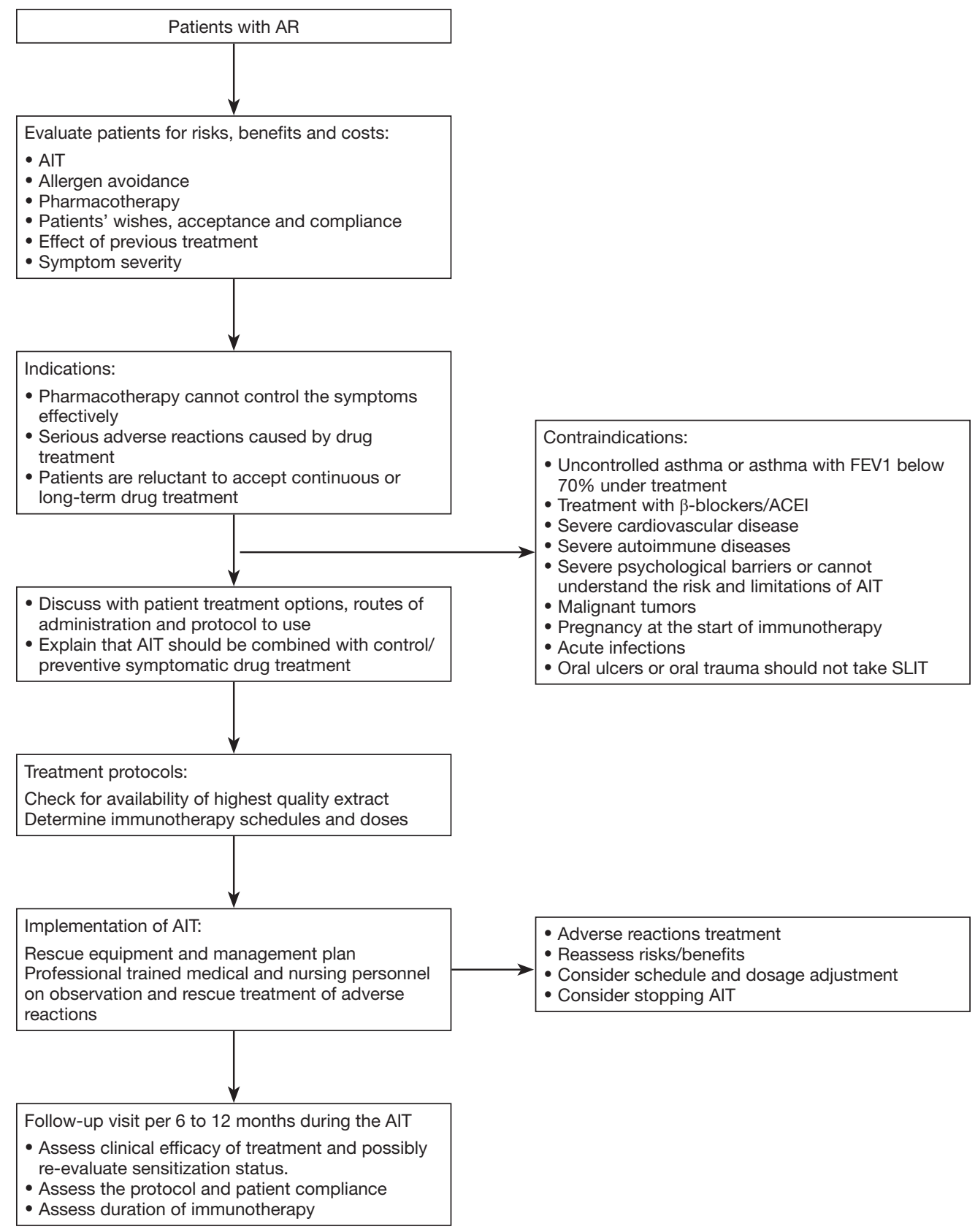

Figure 1 Algorithm and annotations for immunotherapy. AR, allergic rhinitis; AIT, allergen immunotherapy; FEV1, forced expiratory volume in one second.

being the gold standard. The earliest case of sublingual administration of allergen in patients appeared in 1986 (76), and current indications for SLIT are AR (sensitized to pollen allergens, cat fur and HDM) and mild to moderate asthma (sensitized to HDM) (77).

In China, there is only one mite product for use in SLIT at present, and consequently SLIT is not as common as
SCIT in clinical practice. However, it is possible that with the availability of more tried and tested products, SLIT may be more widely used in China in the future.

\section{Other routes of allergen administration in AIT}

Apart from subcutaneous (for SCIT) and sublingual (for 
SLIT) routes of allergen administration, allergen has also been administered via other less common routes; including intra-lymphatically (for intra-lymphatic immunotherapy; ILIT), epicutaneously (for epicutaneous immunotherapy; EPIT), orally (for oral immunotherapy; OIT), nasally (for local nasal immunotherapy; LNIT) and bronchially (for local bronchial immunotherapy; LBIT). ILIT has been shown to reduce the treatment period from $2.5-3$ years to 8 weeks (78), and consequently, this route might become the preferred alternative to conventional SCIT or SLIT. As far as EPIT is concerned, more research is needed to define optimal doses (79). In the case of OIT, its limited efficacy, safety and tolerability are major drawbacks and severely curtailed the use of this technique (80). Similarly, local adverse reactions, requirement of a particular administration technique and effectiveness in rhinitis only has limit the application of LNIT in clinical practice $(81,82)$, whereas LBIT has been abandoned already because controlled trials have failed to demonstrate clinical efficacy and adequate safety of allergen administered via this route (82).

\section{Mechanisms of immunotherapy}

The mechanisms of AIT are still not fully understood (83). AIT is based on administration of gradually increasing concentrations of allergen extracts and leads to the development of clinical allergen tolerance in selected patients. Tolerance is mainly accompanied by the induction of regulatory subsets of $\mathrm{T}$ and $\mathrm{B}$ cells, the production of IgG4 isotype allergen-specific blocking antibodies, and decreased inflammatory responses to allergens by effector cells in inflamed tissues $(46,84,85)$.

During the last 20 years, regulatory $\mathrm{T}$ (Treg) cells have been identified to play a pivotal role in inducing and maintaining peripheral immune tolerance during AIT. Skewing of allergen-specific effector $\mathrm{T}$ cells to a regulatory phenotype is a key event during AIT, not only in the development of healthy immune response to allergens, but also successful outcome in patients receiving AIT. Both sublingual and subcutaneous routes of AIT have been confirmed to induce allergen-specific Treg cells in the clinical setting (86). In addition to Treg cells, other key cell types such as suppressive B cells and dendritic cells (DCs) also appear to play important roles in successful AIT.

\section{Desensitization effects (very early phase) following AIT}

Although relief of IgE-mediated skin sensitivity usually requires more than 1 year of AIT, most patients start to get protection against bee stings or tolerance to skin late-phase response challenges during very early phase following the AIT $(87,88)$. Indeed, a decrease in mast cell and basophil activation and degranulation and systemic anaphylaxis has been found to be initiated from even the first injection (86). However, how AIT mediates this desensitization effect is yet unknown (86-88). In a study investigating oral desensitization in mice, antigen-specific mast cell desensitization was demonstrated to be one of the main underlying mechanisms for oral desensitization (89). Thus, it is possible that in the very early phase, the suppression of mast cells and basophils might be modulated by changes in other immune factors, such as increased Treg cells and specific $\operatorname{IgE}(\mathrm{sIgE})$ levels. One study investigating the protective mechanisms during allergen-specific venom immunotherapy demonstrated that monocytes were activated very early within a few hours after the first injection on day 1 , and that monocyte initiated ILT3/4mediated inhibition, higher IL-10 production, as well as elevated intracellular cAMP might contribute to early induction of protective mechanisms against allergic reactions during the build-up phase of allergen-specific venom immunotherapy (90).

\section{Modulation of antigen-presenting cells (APCs) function following AIT}

APCs, particularly DCs, control both the processes of immune response and peripheral tolerance by means of identification of the environmental signals that are associated with encountered antigen (such as pathogenassociated molecular patterns). The tolerogenic activity of DCs depends on the maturation status and the activation status of the cell, in addition to the cell lineage (for example, myeloid DCs versus plasmacytoid DCs), all of which can be influenced by immunomodulatory agents such as adjuvants (91). In AIT, in the absence of pro-inflammatory signals, airway DCs have an intermediate phenotype between immature and mature; which expresses a range of co-stimulatory molecules resulting in tolerogenic interaction with lymph-node T cells. Following AIT, DCs can induce $\mathrm{T}$ cells with a regulatory phenotype and function, which secrets IL-10. Such Treg cells inhibit subsequent inflammatory responses, which might subsequently lead to the beneficial effects of AIT $(92,93)$. Depletion and adoptive transfer of pulmonary plasmacytoid DCs (pDCs) in a mouse model has demonstrated that pDCs play a central role in 
protection against sensitization to allergen and development of asthma (94). In addition, several clinical trials have shown that APCs; including B cells, monocytes and macrophages; produce increased amounts of IL-10 following AIT, and this might lead to increased generation of IL-10-secreting Treg cells $(95,96)$.

\section{Modulation of T-cell responses and peripheral $T$-cell tolerance to allergens following AIT}

T-cell responses to food allergens and airborne allergens have shown that allergens can induce Th1, Th2 and Treg responses. The ratio of allergen-specific IL-10-secreting cells to IFN- $\gamma$-secreting cells and IL-4-secreting cells determines the development of a healthy or a pathogenic immune response (97). Active regulation might be an essential mechanism for both inducing and maintaining peripheral tolerance to allergens. In allergic diseases, the activity of both allergen-specific IL-10-secreting Treg cells and CD4+CD25+ Treg cells are compromised, but can be ameliorated by AIT (95,97-100).

Modulation of $\mathrm{T}$-cell responses to allergen following AIT can be achieved in several ways, including the following: (I) AIT can increase the allergen-induced ratio of Th1 cytokines to Th2 cytokines $(101,102)$; (II) AIT can induce epitope-specific T-cell anergy that can be blocked by neutralization of IL-10 (103); (III) AIT can generate allergen-specific Treg cells that can suppress the responses of effector $\mathrm{T}$ cells following delivery of either whole allergen or synthetic peptides that contain a T-cell epitope (99); and (IV) AIT can increase the production of cytokines with regulatory activity. Induction of mRNA that encodes IL-10 and increased production of IL-10 protein has been reported to occur in both the blood and the tissues following AIT $(87,99,100,104-106)$. Increased production of transforming growth factor- $\beta$ (TGF- $\beta$ ) has also been reported following AIT, and suggested to contribute to Treg-cell function, because addition of neutralizing TGF $\beta$ specific antibodies to T-cell cultures was found to inhibit the regulatory function of these $T$ cells (100).

\section{Modulation of antibody responses following AIT}

While the level of allergen specific antibody response in healthy control individuals are often too low to be detected, exposure to high concentrations of allergen, has been shown to elevate levels of allergen specific IgG4, IgG1, and IgA, but not sIgE $(99,107)$. Early studies in subjects with allergic conditions indicated that allergen-specific IgG prevented immediate allergic skin inflammation by competing with $\mathrm{IgE}$ for the same epitopes and binding the allergen; thus, being termed blocking antibodies $(108,109)$. However, while the serum concentrations of allergen-specific IgG have been shown to correlate with clinical improvement in some studies, this has not been shown to generally be the case, and thus remains a controversial issue (110-112). Nevertheless, studies analysing the IgG isotypes induced by AIT have shown that the concentrations of IgG1 and IgG4, in particular, were increased 10- to 100 -fold following AIT, likely influencing the blocking of IgE-mediated responses (111,113-115). Indeed, there is sufficient evidence that functional activity, rather than the quantity of $\mathrm{IgG}$ antibodies, might be a more appropriate measure, which seems to correlate more closely with clinical parameters (96). In particular, serum of patients receiving house-dust mite AIT has been shown to contain high levels of specific IgA and IgG4 against Der p1, but not $\operatorname{IgE}(99)$. Furthermore, AIT did not change sIgE after 70 days of treatment. It is important to note that IgA and IgG4 represent noninflammatory isotypes, whereas $\mathrm{IgG} 1$ and $\mathrm{IgE}$ binding to allergens and can initiate an inflammatory response $(99,116)$.

\section{Patient selection}

\section{Indications \& contraindications}

\section{Indications}

AIT has been used to treat allergic diseases in China for many years, and its efficacy confirmed in several clinical studies. AIT is the only treatment which can prevent or change the progression of AR to asthma and whose clinical efficacy can persist for many years after discontinuation of AIT (117). Thus, some international guidelines suggest that AIT can be started sooner if indicated and not only on the premise of drug treatment failure $(118,119)$. Recently published articles have shown that in offspring with one or both parents with allergies, the incidence of allergic diseases was significantly lower when the parents had received AIT treatment than when the parents did not undergo AIT $(120,121)$. In light of these findings, earlier initiation of AIT therapy is indicated for patients who are diagnosed with $\mathrm{AR}$ and whose symptoms are confirmed to be caused by a specific allergen, if appropriate financial and medical resources are available. Standardized allergen agent should be used because the efficacy and safety of AIT depends strictly on the quality of the allergen extract. Based on the 
types of standardized allergen vaccines which are currently available for clinical use in China, we recommend AIT for especially patients sensitized to only a single common allergen such as dust mite, either singly or in combination with no more than 1-2 types of other allergens.

Since AIT is a disease modifying treatment, it should be initiated early in the course of the disease in order to prevent irreversible damage in mucous membranes of the affected organ $(122,123)$. AIT is especially suitable for patients under the following conditions $(124,125)$ : (I) pharmacotherapy (antihistamines, anti-leukotrienes drugs, nasal glucocorticoids, etc.) cannot control the symptoms effectively; (II) serious adverse reaction has occurred during the pharmacotherapy; and (III) patients are reluctant to accept continuous or long-term pharmacotherapy.

As China is a vast country with diverse regions, the level of medical service and resources varies among different regions. Patients' compliance with immunotherapy is also different from one region to another. As patient compliance is key to the success of immunotherapy and also an important factor in avoiding adverse reactions, the following factors should be considered when AIT is recommended (126): (I) patients' preference and compliance; (II) convenience of treatment; (III) severity of symptoms and the effect of drug treatment; and (IV) effects and possibility of allergen avoidance. Before initiating AIT, good communication with the patients is essential, and patients must be informed about the following: the practical procedure, the duration of treatment; the expected effects and potential risks during the treatment. Printed information on how AIT is carried out and how the possible adverse events can be prevented should be available to patients.

SLIT may be considered as initial treatment and failure of pharmacological treatment is not an essential prerequisite for the use of SLIT $(118,119)$. SLIT is also widely used in China, because it is safe, non-invasive, and can be easily administered at home. Many systematic reviews suggest that both SCIT and SLIT are effective for AR $(127,128)$. Although the indication for SLIT is similar to that of SCIT, SLIT is more suitable for patients who cannot tolerate SCIT because of severe adverse reactions, objection for repeated injection, or hospital visits for injection therapy are inconvenient.

\section{Contraindications}

SCIT should be performed only in clinics with resources to manage any possible systemic adverse reactions. Indeed, if possible the patients should be chosen prudently to avoid serious adverse reactions. The major contraindications of SIT (SCIT and SLIT), however, are as the follows (73,124,129):

(I) Patients with severe or uncontrolled asthma (FEV1 $<70 \%$ predicted), and irreversible respiratory obstructive disease. This is a major contraindication for both SCIT and SLIT;

(II) Patients who are using $\beta$-blockers or angiotensin converting enzyme (ACE) inhibitors for treatment. The use of $\beta$-blockers (including surface-absorbing dosage forms) may increase the risk of respiratory adverse reactions, and have an influence on the effect of adrenaline used to rescue severe allergic reactions. The use of ACE inhibitors can inhibit the activation of reninangiotensin system of the human body, and patients are therefore prone to have hypotension shock when allergic reactions occur;

(III) Severe cardiovascular disease. In emergency, patients with severe cardiovascular disease may increase the risk of using adrenaline;

(IV) Severe autoimmune diseases including autoimmune and immunodeficiency diseases such as AIDS, especially for patients during active stage;

(V) Patients with severe psychological barriers or patients who cannot understand the risk and limitations of AIT;

(VI) Malignant tumors. The relationship of allergen vaccine and tumor immunology is not clear, and therefore AIT it is not recommended for patients with malignant tumors;

(VII) Pregnancy. Currently there is no evidence on the adverse effects of immunotherapy on the fetus or pregnant women during pregnancy, however initiation of immunotherapy is not recommended during pregnancy. If the patient is found to be pregnant during the maintenance phase of immunotherapy and tolerates AIT well, then immunotherapy can be continued;

(VIII) Several special conditions (130): It is not recommended for seasonal AR (hay fever) patients to start immunotherapy during the pollen spread period. The AIT injection should be suspended if the patient has an acute infection or fever, or is taking other types of vaccines. In addition, on the day of injection, the patient should avoid any factors that may promote allergic reactions, such as strenuous exercise, alcohol, etc.; 
(IX) Patients with oral ulcers or oral trauma should not be prescribed SLIT.

\section{Special considerations in immunotherapy}

\section{Special considerations in children}

The HDM is the most prevalent allergen in patients with asthma and/or rhinitis in China (20). A cross-sectional study in China, involving 6,304 patients suffering from asthma and/or rhinitis, indicated that $72.1 \%$ had at least one positive skin reaction; with $59.0 \%$ prevalence for $\mathrm{Der}$ $f$ and $57.6 \%$ prevalence for Der $p$. Furthermore, children had significantly higher positive prevalence rates to mites than adults; with male children demonstrating significantly higher prevalence than female children in all the regions of the country investigated (20).

Age and safety are important factors to consider when initiating AIT treatment. Although AIT has been proven to have a protective effect against development of asthma in children with AR, children younger than 3 years are seldom involved in clinical practice and trials, due to their poor compliance and difficulty in communicating the benefits and possible risks of AIT. The recommended optimal duration of AIT to achieve maximal efficacy with safety for AR in children is presently unclear. However, one recent study in China has demonstrated good tolerance and safety of SLIT in children as young as 3 years old with $\operatorname{Der} f$-induced AR with or without asthma (54). Similarly, another study has reported good tolerance and safety of HDM-SCIT in over 5 years-old children with a history of HDM-induced AR or asthma (47).

Several studies have reported SLIT and SCIT to be efficacious and safe in the treatment of allergic diseases (AR, allergic asthma) in Chinese children $(46-48,131,132)$. A real-world, multicenter, single-arm, open-label study showed that SCIT with semi-depot HDM allergen extract was safe and effective in the treatment of AR and asthma in a Chinese population (47). Adverse events occurred with $1 \%$ of all injections, and study related adverse events occurred in $16.9 \%$ of all subjects; although no serious adverse events or serious systemic allergic reactions were reported (47). Another study suggested that SLIT with HDM extract was also effective and safe for children with AR (48). One retrospective study compared the efficacy and safety of SLIT and SCIT in 186 children with AR sensitized to HDM (131). After a 2-year treatment, both SLIT- and SCIT-treated groups showed significant reduction in total rhinitis symptoms score (TRSS), VAS, the rhinoconjunctivitis quality of life questionnaire (RQLQ), skin index (SI) and eosinophil cationic protein (ECP) compared with baseline. The incidence of adverse reactions reported with SLIT and SCIT were $3.85 \%$ and $2.44 \%$, respectively. In contrast, one study reported that $17.0 \%$ of asthmatic children receiving HDM-SCIT had systemic adverse events, of which 5.4\% were severe [local reactions (LRs) with wheezing and peak expiratory flow (PEF) decrease] but could be easily resolved (132). However, no life-threatening events were reported in these children. Despite these grouped findings, evaluation of the benefits/risks ratio and compliance in individual patients is of critical importance, before a decision is made to proceed with AIT. Moreover, standardized drug treatment should be prescribed in combination with AIT to ensure good disease control and reduce the risk of adverse effects. Also, AIT should never be recommended as a monotherapy for asthma.

Another recent retrospective study evaluated the efficacy of SLIT with standardized Der $f$ drops in 162 children (aged 4-12 years) with AR, who were mono-sensitized or polysensitized according to SPT results (133). The efficacy and safety were evaluated before treatment, 2 years after SLIT initiation and 3 years after drug discontinuance. The authors found that SLIT had a long-term efficacy in both mono-sensitized and polysensitized patients with $\mathrm{AR}$ and that a longer SLIT treatment ( $>2$ years) may be necessary to consolidate its efficacy (133). Similarly, Li and colleagues (134) investigated the effect of SLIT for 1 year in 112 children with HDM-induced respiratory allergic disease, allocated to a mono-sensitized- or a polysensitized-group, and demonstrated that there was no significant difference in the clinical effects between polysensitized and mono-sensitized children.

Polysensitized asthmatic children are common in clinical practice. However, not every sensitizing allergen is relevant to the symptoms; and thus, selection of allergen for AIT should be based on the corresponding results tests of sensitization and effects on symptoms. SPT should be performed before the first dose of AIT to evaluate the response severity, and avoid administering allergen extracts to individuals who might possibly experience anaphylactic reactions. Administration of different allergen extracts at different times of a day or different locations is recommended if AIT must involve multiple allergens (135).

AIT requires good compliance to maintain the longterm administration of allergen extracts. Children's characteristics, adherence to treatment, and especially the route of administration of allergen extract should be taken 
into consideration before initiating AIT. Patients' education could also be another driving force to encourage children to stick to the therapy.

\section{The use of SCIT during pregnancy}

Several studies from around the world have assessed the safety of SCIT during pregnancy (136-139). An early study by Metzger and colleagues (136) observed 90 atopic women (mostly AR and asthma) who had undergone SCIT during one or more pregnancies, for a total of 121 pregnancies, and demonstrated that the incidence of prematurity, toxemia, abortion, neonatal death, and congenital malformation was no greater than that for the general population. Furthermore, the offspring of the treated mothers developed allergic disease as frequently as children born into allergic families. These findings for no difference in the incidence of abortion, perinatal mortality, prematurity, toxaemia and congenital malformation in women receiving SCIT during pregnancy and in the general population has been confirmed in several subsequent studies $(137,138)$. Indeed, SCIT was found to be safe even when initiated for the first time in a pregnant patient and continuation of SCIT during pregnancy was not correlated with any increased risk to the mother or fetus (137). Thus, it is recommended that maintenance SCIT may be continued during pregnancy $(27,139)$. However, data concerning the use and safety of continuation of SCIT during pregnancy is presently lacking in China. Indeed, in China, before initiation of SCIT, it is usually cleared whether the patient has a plan for pregnancy in the near future. Also, because of the particularity of Chinese medical regulations patients with any plans for pregnancy are temporarily not treated with SCIT. In future, the number of SCIT cases during pregnancy should be gradually increased to acquire more information and experience about Chinese.

In view of the availability of relatively few data on the safety of initiating SCIT during pregnancy, initiation of SCIT should not be encouraged during pregnancy, especially in the high-risk scenarios such as women with prior anaphylaxis to Hymenoptera venom (27). In China, given the limitation of medical environment and patients' recognition, it is recommended that SCIT should not be initiated during pregnancy.

\section{Allergen extracts}

\section{Allergen extracts and allergen standardization}

Confirmation of sensitization to specific allergens in allergic patients and subsequent successful and safe outcome of specific AIT depend on the use of high quality allergen extracts (140). An allergen extract is prepared from natural source materials; e.g., mites, pollen, animal dander, molds; and mainly contain active substances (i.e., proteins or glycoproteins, and non-allergenic molecules). These active substances are allergens which elicit an IgEmediated response in the human immune system (141). The concentration and composition of individual allergens in the extracts may vary significantly, and standardization of the extract is thus a prerequisite in controlling variability and achieving consistency and reproducibility in diagnosis and specific immunotherapy of allergic diseases (142).

Similar to European countries (140), certified references for allergen standardization are not available in China. In-House Reference (IHR) is used for standardization by individual manufactures (143) and labeled in manufacturerspecific units. Each batch of the allergen product is compared to a respective IHR. The IHR must be well characterized by a series of in vitro methods. The total biological potency is generally determined by an in vivo method. Subsequent batches of allergen extract are compared with the IHR and thus the in vivo method is not necessary in batch-to-batch standardization. Currently only HDM allergen extracts (from either $\operatorname{Der} p$ or $\operatorname{Der} f$, and mixtures of the two species) are available and authorized in China for use in vivo allergy diagnosis and specific immunotherapy (31); likely because $\operatorname{Der} p$ and $\operatorname{Der} f$ are the two most common sensitizing allergens in Chinese allergic patients (20) and they are found in most beddings throughout the country (144). HDM allergen extracts are produced as aqueous, glycerinated and aluminumprecipitated formulations for the Chinese AIM market. Standardized products are labeled in biological units $(1,145)$, which currently appear on labels of marketed products as SQ-U/mL, TU, and HEP. Non-standardized allergen extracts are labeled as wt/vol (weight in grams per volume in millilitres). Extracts with a particular wt/vol may have wide variability of biological potency $(146,147)$.

China is a vast country, with a large population and botanically and ecologically diverse regions. Consequently, apart from HDM allergens, patients in different parts of the country are also sensitized to other commonly occurring allergens such as animal dander $(20,148,149)$, weed pollen (150-153), tree pollen (154-156), grass pollen (157), etc. Thus, in view of current availability of only commercial HDM products for AIT in China, there is urgent need for standardised commercial preparations of other specific 
allergen extracts for AIT. Although a few inhaled and food allergens are marketed for in vitro IgE testing, most allergen extracts are not yet standardized.

Presently, the China Food and Drug Administration (CFDA; http://www.sda.gov.cn/WS01/CL0237/15710. $\mathrm{html}$ ) provides guidance and regulates the requirements for manufacture, quality control and characterisation of allergen products in China. This includes the requirements for sourcing and initial control of raw material of specific allergens, including pollen, fungi, mites, animal-borne and food; techniques employed for harvesting, pretreatment, storage, and extraction of allergen; the stages of the production process such as crushing, extraction, filtration, purification, dialysis, concentration, separation, sterilization and lyophilisation; quality control of allergen vaccine, including assessment of total protein content, protein composition, major allergen contents, total allergenic potency, stability, etc.; and standardisation of the extract according to well-defined, fully identified and stable internal reference (IHR) substance and/or International Standards (IS). Assessment of protein composition ensures the presence in the final product of all allergens present in the source material $(142,158)$. It can be measured with sodium dodecyl sulfate polyacrylamide gel electrophoresis (SDS-PAGE), crossed immune-electrophoresis (CIE), or isoelectric focusing (IEF). CIE offers an advantage as a semi-quantitative technique and ensures all major allergens constantly precipitate on agarose gels $(159,160)$. Mass spectrometry is a more powerful tool for analysis of allergic proteins, including detection of isoforms (140), but the method has not yet been applied in China. Quantification of major allergens ensures that the essential allergens are present in a constant ratio (158). The major allergens can be identified using crossed radio-immunoelectrophoresis (CRIE) and the constituents of the major allergens may be determined by quantitative immunoelectrophoresis (QIE) and enzyme-linked immunosorbent assay (ELISA). Quantification of allergenic potency ensures that the overall biological activity of the allergen extract is constant $(142,158)$. Allergenic potency can be measured by either skin tests (SPTs and intradermal tests) or by in vitro techniques, i.e., radio allergosorbent test (RAST) or RAST inhibition assays. The variation in allergenic potency must be within a $50-200 \%$ interval of the stated value according to CFDA requirements.

For modified allergen products, e.g., denatured or chemically modified allergoids, quality control can be performed at the intermediate stages. For an allergen which has IS, a comparison of IHRs and IS should be performed qualitatively and quantitatively. The potency of IHR can be calibrated using the IS value if the potency curves are parallel. However, the CFDA guidance does not provide requirements for standardization of systems used by different manufactures as different methods and processes are applied by different manufacturers. Thus, quality control may assure consistency of batches from one manufacture but does not provide comparability of products from different manufactures (161). Although allergenic products have been standardized by different methods and the quality of extracts on the market varies significantly, only products with specified allergenic potency and concentrations of individual allergens can be used for allergy diagnosis and specific immunotherapy (145).

In contrast to allergen extracts from natural sources, recombinant allergens are generated using recombinant DNA technology and their quality depends on cell lines used, fermentation processes and purification procedures. Recombinant allergen consists of predefined allergenic polypeptides. The quantity and structure of the polypeptides should be determined. Although some recombinant allergens have comparable allergenic activities to natural proteins (162), the regulatory authorities will likely not recognize previous documentations based on natural allergens. Market authorization must rely on the documentations of safety and efficacy obtained during the development of the recombinant products (142). The recombinant allergens from $\operatorname{Der} f$ have been investigated extensively in China (163-171); However, there is no information on recombinant allergens in CFDA guidance and no recombinant product has been authorized yet.

In summary, standardization of allergen products to be used for AIT is necessary to ensure consistency and reproducibility of the efficacy and safety of the product in the management of allergic disease. However, presently there is no uniform standardization system in China and the quality of allergenic products on the market varies significantly. Consequently, we recommend using products with specified total potency and concentrations of individual allergens for AIT.

\section{Skin tests and sIgE antibody tests}

\section{Skin tests}

Skin tests include SPTs and intradermal tests. SPTs are widely used to detect immediate IgE-mediated allergic 
Table 1 Differences between SPTs and serum specific IgE tests

\begin{tabular}{lll}
\hline Variables & SPTs & Serum specific lgE tests \\
\hline Theory & Interaction between antigen and antibody & Immunoassay of allergen-specific IgE \\
Sensitivity & Higher & High \\
Specificity & High & Higher \\
Drug effects & $\mathrm{H}_{1}$-antihistamines drugs have suppressive effect & No effect \\
Skin & Healthy skin required & No requirement \\
Results interpretation & More subjective & More objective \\
Risks & Have certain risks & No risks \\
Cost & Low & High \\
\hline
\end{tabular}

SPTs, skin prick tests; IgE, immunoglobulin E.

reactions, and demonstrate high sensitivity and specificity during the diagnosis of inhalant allergens $(1,172,173)$. Unfortunately, the clinical value of intradermal tests for the diagnosis of inhalant allergens is unclear and thus they are not much used in the diagnosis of inhalant antigen. Intradermal tests are also less safe to perform.

Standardized allergen extracts, negative (saline) and positive $(10 \mathrm{mg} / \mathrm{mL}$ histamine dihydrochloride solution, or $1.70 \mathrm{mg} / \mathrm{mL}$ histamine phosphate solution) controls are required for SPTs. Wheal or erythema is measured around 15 to 20 minutes after the administration of the extracts. Wheal diameters $\geq 3 \mathrm{~mm}$ are considered positive in SPTs. SI is often used to assess the results of SPTs. SI is the ratio of average diameter of allergen to positive control, and determined according to 4 grades:,$+ 0.3 \leq \mathrm{SI}<0.5 ;++, 0.5 \leq$ SI $<1.0 ;+++, 1.0 \leq$ SI $<2.0 ;++++$ SI $\geq 2.0$.

The diagnosis of allergic diseases is dependent on the quality of allergen extracts used in the tests. Currently, several kinds of allergen extracts are available from Wolwo, ALK and NHD in China. The panel of allergens used in the tests depends on the allergens to which the patient has been exposed. Although dust mite is the most prevalent allergen in China, the distribution of other allergens has obvious regional characteristics (174). Care should be taken when SPTs are performed: (I) standardized allergen extracts should be used; (II) positive and negative controls are required; (III) tests should be performed on healthy skin, and areas with widespread urticarial or eczema should not be used for allergen testing; (IV) the patient should be evaluated for dermographism at the same time; (V) it is always necessary to ask patients about the medication they have taken, because drugs such as $\mathrm{Oral} \mathrm{H}_{1}$-antihistamines can suppress SPTs, and the suppression can last for 2-7 days; (VI) the diameter of the largest wheal should be measured.

A patient's medical history and clinical symptoms should be considered when interpreting the SPTs results. Falsepositive skin tests can result from dermographism or can be caused by a nonspecific enhancement from a nearby strong positive reaction. False-negative skin tests may also be caused by the use of extracts of poor initial potency, drugs modulating the allergic reaction, or improper technique. Hence, SPTs should be carried out by trained health professionals.

\section{sIgE antibody tests}

Serum $\operatorname{sIgE}$ antibody tests can be used in patients of any age, especially elderly people who are more likely to produce negative skin tests (175). Consequently, serum sIgE tests are of great importance in the diagnosis of allergic diseases. Normally, low levels of serum sIgE are less likely to produce symptoms than higher levels, but this is not the case with allergic symptoms (176), particularly in very young children. Serum sIgE antibody tests are particularly helpful when the SPTs are negative and there is a high clinical suspicion for AR $(177,178)$. The differences between SPTs and serum sIgE tests are listed in Table 1.

The levels of serum sIgE antibody are graded as follows: grade $0,<0.35 \mathrm{kU} / \mathrm{L}$; grade $1,0.35-0.69 \mathrm{kU} / \mathrm{L}$; grade 2, $0.7-$ $3.4 \mathrm{kU} / \mathrm{L}$; grade 3, 3.5-17.4 kU/L; grade 4, 17.5-49.9 kU/L; grade $5,50-100 \mathrm{kU} / \mathrm{L}$; grade $6,>100 \mathrm{kU} / \mathrm{L}$.

\section{Establishment and administration of immunotherapy centers}

Due to the unique characteristics and safety concerns 
inherent in specific immunotherapy, treatment centers that provide immunotherapy need to establish standardized administrative systems. The aim of this chapter is to describe suitable administrative norms for AIT centers in China.

\section{Locations}

The centers providing AIT should have units comprising

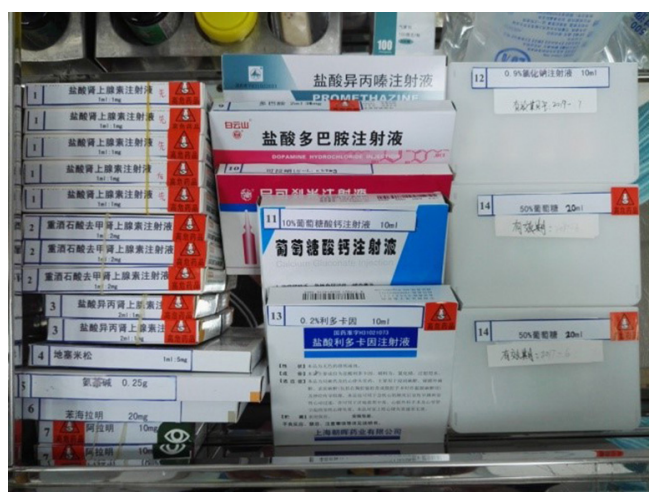

Figure 2 Drugs in the crash cart for emergency use. designated areas for consultation/examination, a laboratory for skin prick testing, respiratory physiology and immunologic assessment, allergen vaccine injection and treatment, patient observation, an emergency treatment and a medical documentation storage. These rooms/ laboratories should be allocated according to the specific conditions prevailing at each institute. The physician consulting room should be equipped with the necessary examination instruments, and should be as clean, well-lit, and spacious as possible. The SPT laboratory should be equipped with a $2-4^{\circ} \mathrm{C}$ refrigerator to store the necessary allergen skin test reagents, and also have sufficient illumination and space. The injection and treatment room must also be equipped with a $2-4{ }^{\circ} \mathrm{C}$ refrigerator to store allergen vaccines; all refrigerators must be equipped with thermometers to record refrigerator temperatures on a daily basis. The observation room should be across from the injection and treatment room, with a sufficiently wide passageway or a sufficiently large glass pane to allow observation of patient responses. The emergency room should be next to the observation room and equipped with all drugs and medical equipment/instruments necessary for rescue in an emergency (Figures 2 and 3).
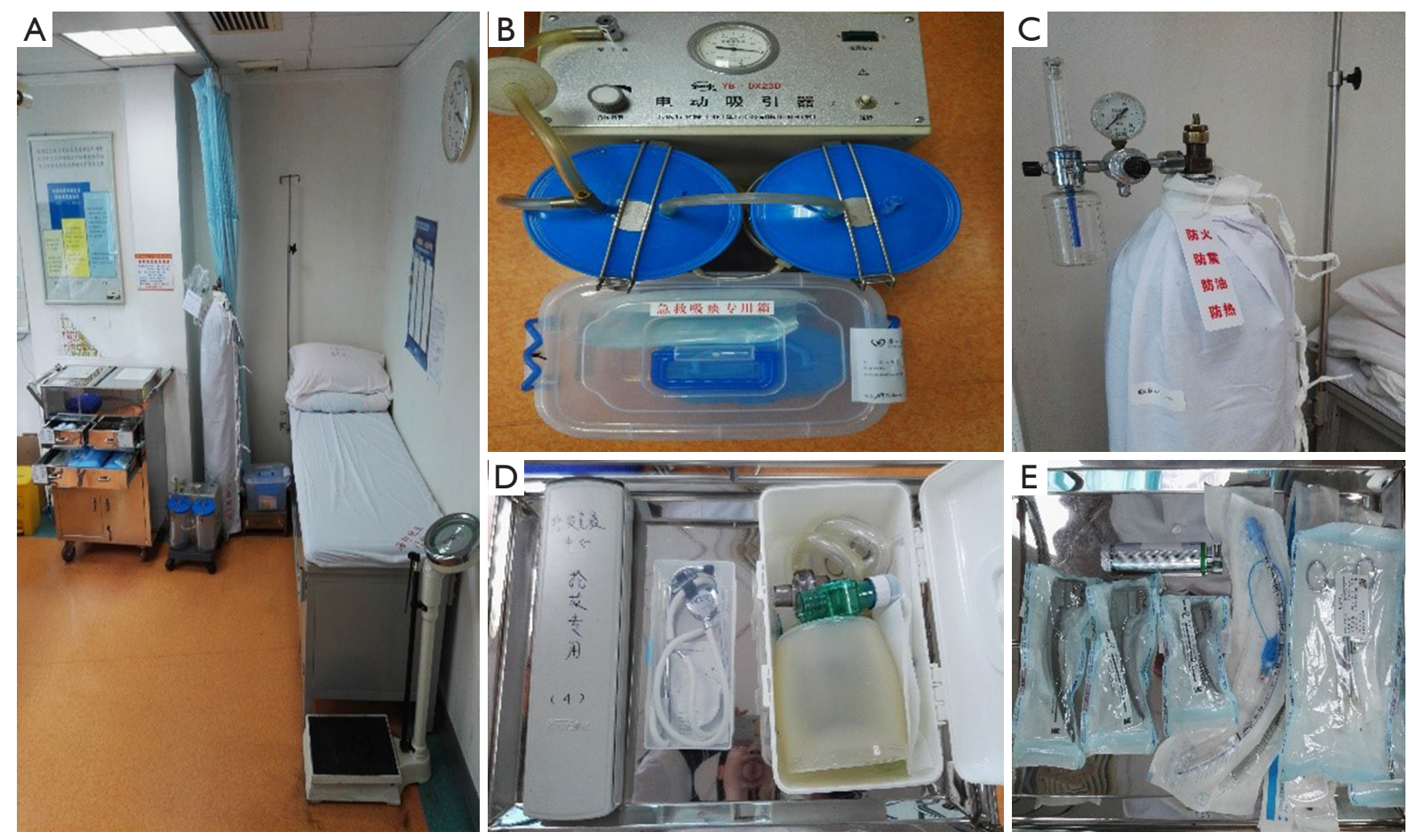

Figure 3 Essential emergency equipment in immunotherapy centre. (A) The emergency room bed; (B) the mucus suction pump; (C) the oxygen tank; (D) bag valve mask, blood pressure meter and stethoscope; (E) the endotracheal tube. 


\section{Allocation of institutional personnel}

Institutions administering AIT should have a managing director and specialized physicians who have undergone professional training in the field of AIT. Furthermore, there should be at least three specialist trained nurses, respiratory function and immunological laboratory technicians, and administrators filing and storing all patient data.

\section{Administration of immunotherapy center}

\section{Qualifications and duties}

A physician specializing in allergies (or is qualified in allied medical fields) should be in charge of the immunotherapy institution. This person should specifically have a senior professional title in related specialties such as allergies; respiratory medicine; otorhinolaryngology head and neck surgery; pediatrics; and dermatology. The person in charge shall be fully accountable for the implementation of SCIT and safety measures. The physician undertaking specific immunotherapy in children should be a specialist with appropriate knowledge in paediatrics, particularly pediatric allergic conditions. The person in charge of the center should ensure that staff members have been trained and can perform their tasks professionally and appropriately. All staff members administering SCIT should have welldefined duties, which are formulated with full consideration of local principles and actual conditions.

\section{Training of immunotherapy personnel}

Medical and nursing personnel must undergo regular training and attend continuing educational courses. Training content should include the following: (I) evaluation of patient conditions from the perspective of receiving SCIT (including clinical conditions and testing of peak respiratory flow) to determine when injection of allergen may begin or continue at the current dose. Parents/guardians of patients under 15 years of age must be involved in the patient evaluation process; (II) recording of data and information in each patient's immunotherapy records; (III) injection techniques; (IV) dose adjustments; (V) strict observation of patient conditions (including adult and pediatric patients); (VI) discovery of adverse reactions as early as possible; (VII) treatment and monitoring of patients who present with adverse reactions; (VIII) implementation of regular re-examinations or therapeutic evaluations; (IX) factors that influence the decision to continue or terminate AIT treatment.
Each year, the center should organize anaphylactic shock rescue training for its subordinate medical and nursing personnel, including the emergency procedures and correct usage of emergency rescue equipment. Records of training should be maintained.

Prior to performing their work, medical and nursing personnel must undergo strict training in cardiopulmonary resuscitation techniques. They should be familiar with operational techniques; including cardiopulmonary resuscitation, endotracheal intubation, use of manual resuscitation airbags, sputum suction, oxygen inhalation, and aerosol inhalation. Medical and nursing personnel should be proficient in their grasp of the rescue procedures for anaphylactic shock. Technical training for cardiopulmonary resuscitation should be conducted annually; and the hospital should be responsible for carrying out spot examinations, recording the results, and conducting unified management.

\section{Procurement and storage of allergen vaccines}

Allergen vaccines should be uniformly procured by the hospital, using cold chain transportation methods. Standard procedures should be utilized jointly with the pharmacy to ensure refrigeration conditions, and freezing would not be permitted. The vaccines should be stored in $2-8{ }^{\circ} \mathrm{C}$ refrigerators away from direct sunlight, and may be stored for 6 months after opening.

\section{Equipment and environment within the centre}

Because treatment may induce allergic reactions, the institution must be stocked with suitable drugs and equipment for responding to emergency events such as anaphylactic shock.

\section{Emergency drugs}

Adrenaline hydrochloride, norepinephrine, isoproterenol, dexamethasone, aminophylline, diphenhydramine, aramine, phenergan, $0.2 \%$ lidocaine, $50 \%$ GS, $10 \%$ calcium gluconate, vantorin aerosol, coramine, sodium chloride injection, $5 \%$ GS, $10 \%$ GS, and 5\% GNS should be stocked for all emergency events (Table 2 and Figure 2).

\section{Emergency equipment}

Bag valve mask, mucus suction pump, oxygen supply, endotracheal tube, anesthetic laryngoscope, scissors, speculum, blood pressure meter, stethoscope, syringe and needle, iodine disinfectant, cotton swabs, tape, opener, and tourniquet should be readily available (Figure 3). 
Table 2 List of drugs, dosage and indications for emergency events

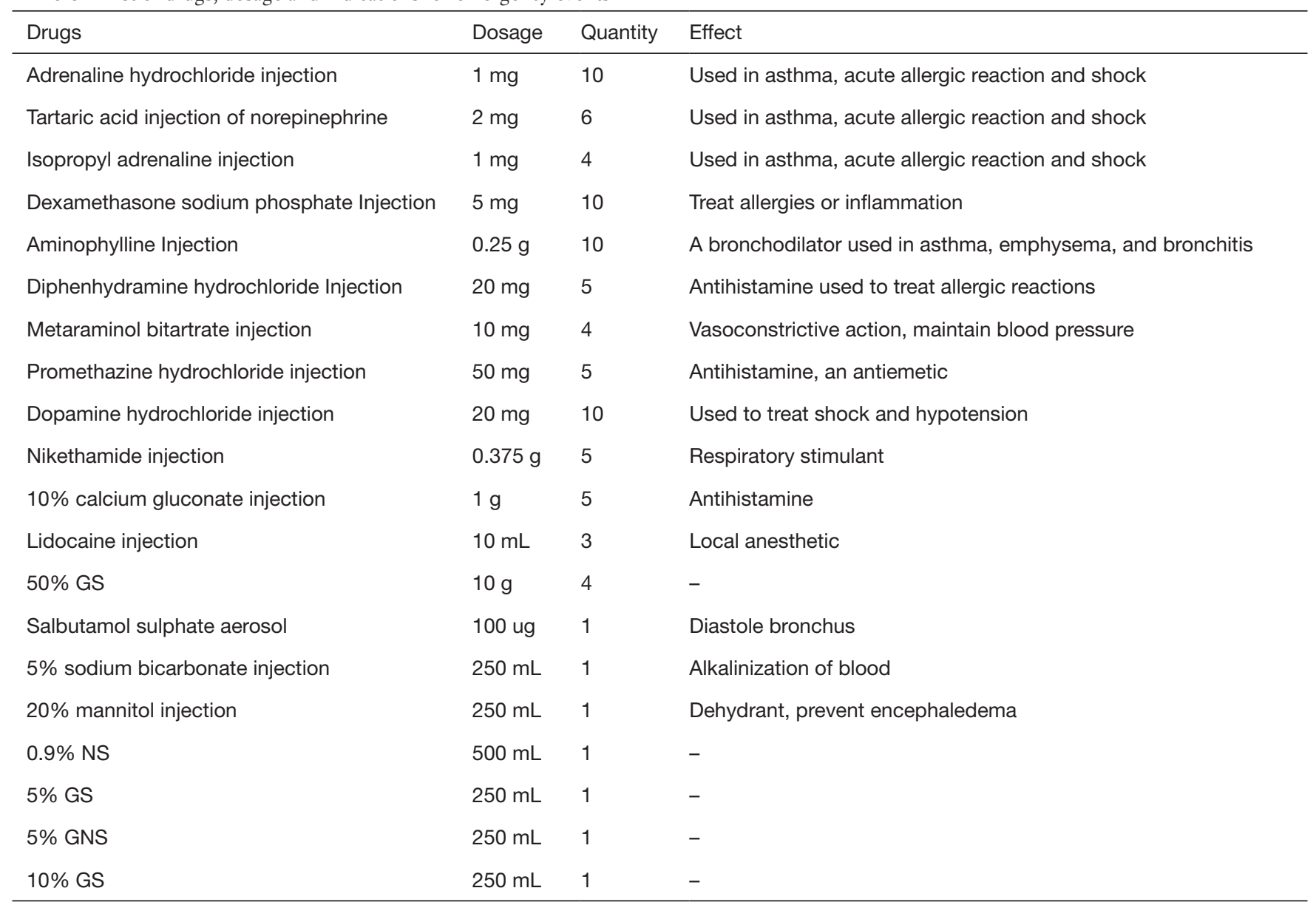

GS, glucose saline; NS, normal saline; GNS, glucose solution.

\section{Management system for emergency drugs}

(I) Emergency drugs must be placed in designated areas or locations such as the crash cart or specialized crash cabinet;

(II) Emergency drugs must be stored with clear labels covering types and characteristics (such as injection, ingestion, topical application, or toxic drugs);

(III) The types and quantities of emergency drugs must satisfy clinical emergency treatment needs;

(IV) Emergency drugs must be kept under lock and key by specialized personnel and may not be removed or used without authorization. The emergency drug register should include information on the names, specifications, quantities, and expiration dates of emergency drugs for reference at any time. There should be a list of emergency drugs in the emergency cabinet;

(V) A system for inspection of emergency drug base number of quality should be established. The emergency drug register should be placed outside crash carts. The emergency drugs and their specifications, types, quantities, and expiration dates should be checked daily for consistency with the register, and each item recorded and signed for. The daily records in the crash carts should be exhibited clearly;

(VI) After each usage, the emergency drugs must be restocked in a timely manner. On the following day, the team leader in charge for the shift should confirm the inventory to ensure that the emergency drugs are readily available for use at any time for emergencies;

(VII) Medical and nursing personnel must be familiar with the configurations of the center's emergency drugs and instruments, shall follow placement rules, and check their quantities and use on a daily basis to ensure that all the emergency equipment is in a fit state ready for use. 


\section{Standards for environmental controls}

Pursuant to the hospital's hygienic standards, each month, the hospital shall test, sample, and record the hand hygiene of medical and nursing personnel; each quarter, the hospital shall test the irradiation intensity of ultraviolet lamps in the consulting and treatment rooms, for timely replacement if $<70 \mu \mathrm{W} / \mathrm{cm}^{2}$; records shall be properly maintained.

Total number of surface bacterial colonies detected shall be $\leq 10 \mathrm{cfu} / \mathrm{cm}^{2}$ in the treatment and injection rooms, and antiseptic hand rub shall be provided.

\section{Management of patient documentation and materials}

The institution shall establish a set of documentation records for each patient to include general information on the patient's physical history, family history, personal history, and history of allergies; the signed informed consent form; information on drugs administered before, during, and after treatment; results of allergen tests, pulmonary ventilation function and airway responsiveness tests, and other laboratory tests; symptoms scores before and following drug administration; immunotherapy vaccine doses and timetables; and records of adverse reactions. Patient documentation should be done according to a unified numbering system and records should be stored in a data room in a uniform manner under specialist management. Qualified institutions may utilize digitized documentation management systems.

\section{Appointment system for patients}

The institution should possess a list of all patients undergoing immunotherapy, marking the patients' names, telephone numbers, and planned visits at the institution. Specialists should be responsible for making appointments with patients for immunotherapy and for rebooking any missed appointments.

\section{Information for patients and their rights}

Patients should receive instruction before and during treatment regarding the treatment period so that they fully understand the purpose and process of specific immunotherapy, as well as potential adverse reactions, while at the same time stressing the importance of symptomatic drug treatment (particularly in the early stages). In particular, patients should understand that they need to report their physical responses in a timely manner during the observation period after vaccine injection in order to enhance safety and minimize the risks of immunotherapy.

Prior to the commencement of specific immunotherapy, the patient or the patient's guardian must sign the informed consent form. The informed consent form shall include details on the principles of specific immunotherapy, the immunotherapy treatment process, and possible adverse reactions and emergency treatment measures to be applied. The consent form shall also include details of the patient's disclosure of history of allergies, authorization for emergency treatment by physicians in the event of adverse reactions, and guarantee to bear any expenses arising from emergency treatment.

\section{Immunotherapy schedules and doses}

SCIT generally consists of two phases: a build-up phase (also known as up-dosing or induction) and a maintenance phase (27). The conventional schedule of SCIT consists of a dose build up by means of one-weekly injections for about 15 weeks, followed by maintenance dose injections at 4- or 8 -week intervals for 3 years (26). The build-up phase can be shortened by cluster or rush schedules. During the cluster schedule, multiple injections (usually 2-3) are administered in one visit per week and reach maintenance dose within a few weeks. During the rush protocol, multiple injections are administered on consecutive days, and reach maintenance dose typically within one week. It has been shown that following a cluster schedule, there is a more rapid achievement of symptomatic improvement, with no increase in systemic reactions (SRs) $(26,179)$. In contrast, a rush protocol is associated with an increase in SRs sometimes, however, these can generally be well tolerated $(180,181)$.

The majority of patients with AR or allergic asthma are polysensitized. Some studies have shown that AIT is equally effective in mono-sensitized and polysensitized patients, when clinically relevant allergens are selected for AIT (133-135). The majority of the published randomized controlled studies of both SCIT and SLIT have been conducted with single allergen extracts, and also there is conflicting evidence for the effectiveness of allergen mixes (124). Whilst simultaneous delivery of multiple unrelated allergens may be clinically effective, there is a need for additional investigation of therapy with more than 2 allergen extracts (particularly in SLIT). Furthermore, SCIT or SLIT with multiple allergens in polysensitized patient needs more 
supporting data from large scale clinical trials to validate this as a treatment option (182).

\section{Conventional immunotherapy}

The main forms of immunotherapy currently employed in China are SCIT and SLIT. Although subcutaneous injection has been the predominant method of allergen administration, sublingual application of the extracts has increased over the last two decades and is now the dominant approach in several European countries (183). Additional approaches to ASIT under active investigation include epicutaneous and intra-lymphatic administration $(184,185)$.

\section{Starting dose}

Initial dose of immunotherapy is dependent on the method used for allergy testing. In order of precision, intradermal dilutional testing (IDT), in vitro serologic testing, IDT with extrapolation, modified quantitative testing (MQT) or blended techniques, and skin prick testing can all be used to estimate the starting dose of immunotherapy. In IDT based immunotherapy, the same extract that is used for testing is also used for treatment. IDT-based immunotherapy also determines a patient's level of sensitivity to each antigen (186). Immunotherapy begins at a time when symptoms are as mild as possible and treatment is always initiated with the lowest dose of the weakest strength. With highly sensitive patients, specific immunotherapy is carried out in accordance with the "highly sensitive" dosage guidelines and also started with strength $1: 10$ of the initial dose.

\section{Frequency of build-up injections}

The conventional schedule for SCIT with unmodified allergen extracts consists of a dose build up by means of oneweekly injections, followed by maintenance dose injections at 4- or 8-week intervals. Fewer build-up injections are possible with modified allergenic extracts, such as allergoids or addition of adjuvants (124). The injections of gradually increasing doses should be administered at 7-day intervals. While the interval between any two injections must never be less than 7 days, an increase in the injection interval to up to 14 days is acceptable. Dose increases must be made cautiously, especially in highly sensitized individuals, using intermediate dose levels if necessary, until the patient's individual tolerance limit is reached. A patient's tolerance limit is the individual maximum dose and must never be exceeded to avoid the risk of allergic side reactions.

\section{Reductions in dose during periods of exacerbation of symptoms}

If the current injection is well tolerated, then the dose is increased step by step to the individual maximum dose (the maintenance treatment dose). However, if the previous injection has not been well tolerated, then the subsequent dose is either reduced to the last well-tolerated dose (for intense LRs) or reduced by 2-3 steps (for mild SRs). For severe SRs, immunotherapy is either restarted from starting dose or discontinued (47).

\section{Dose adjustments for late injections}

If initial AIT in the build-up phase is interrupted for more than 2 to 4 weeks after the last injection, therapy should be continued at half the last dose as a safety precaution. If therapy has been interrupted for more than 4 weeks, then it must be re-initiated at the lowest dose of the weakest strength.

When starting a new pack of doses for maintenance treatment, the first dose taken from the pack must not exceed $20 \%$ (mites: 50\%) of the last dose the patient has received. The dose can then be increased again to the individual maximum dose (at intervals of 7 to 14 days), with interim stages according to the degree of sensitivity and prior tolerance of the treatment.

During maintenance treatment, if the scheduled injection is delayed for up to 2 weeks, then therapy must be continued at no more than half the last administered dose as a precaution. If the scheduled injection time is exceeded by more than 2 weeks, then treatment must be resumed at no more than $5 \%$ of the last administered dose. With an interruption of more than 1 year, the treatment must be restarted from the beginning.

\section{Maintenance schedules}

With non-seasonal allergens (e.g., mites), the individual maximum dose achieved is administered as booster injection during the whole year (after a gradual extension of the injection intervals) at approximately 4- to 6-week intervals.

The customary duration of ASIT is 3 to 5 years, although duration of 3 years for both SCIT and SLIT is recommended in the treatment of AR. However, evidence from a long-term open controlled study suggests that a 3-year course of SLIT might not be sufficient for longterm protection (187). On the other hand, prospective studies of SCIT with grass pollen extract for AR and HDM extract for asthmatic patients suggest that 3 years of ASIT produces prolonged remission of symptoms after discontinuation $(188,189)$. 
Table 3 Dosing schedules of dermatophagoides farinae preparation

\begin{tabular}{|c|c|c|c|c|c|}
\hline Day & $\begin{array}{l}\text { The first week, } \\
\text { No. } 1(1 \mu \mathrm{g} / \mathrm{mL})\end{array}$ & $\begin{array}{l}\text { The second week, } \\
\text { No. } 2(10 \mu \mathrm{g} / \mathrm{mL})\end{array}$ & $\begin{array}{l}\text { The third week, } \\
\text { No. } 3(100 \mu \mathrm{g} / \mathrm{mL})\end{array}$ & $\begin{array}{l}\text { The fourth week } \\
\text { No. } 4(333 \mu \mathrm{g} / \mathrm{mL})\end{array}$ & $\begin{array}{l}\text { The fifth week, } \\
\text { No. } 5(1,000 \mu \mathrm{g} / \mathrm{mL})\end{array}$ \\
\hline 1 & 1 drop & 1 drops & 1 drop & \multirow{4}{*}{$\begin{array}{l}3 \text { drops each day for a dose } \\
\text { maintenance (age }<14 \text { years); } \\
3 \text { drops each day for } 1 \text { week } \\
\text { (age } \geq 14 \text { years) }\end{array}$} & \multirow{4}{*}{$\begin{array}{l}2 \text { drops each day for a } \\
\text { dose maintenance (age } \\
\geq 14 \text { years) }\end{array}$} \\
\hline 3 & 3 drops & 3 drops & 3 drops & & \\
\hline 6 & 8 drops & 8 drops & 8 drops & & \\
\hline 7 & 10 drops & 10 drops & 10 drops & & \\
\hline
\end{tabular}

\section{SLIT in China}

Dermatophagoides farinae preparation is the main allergen extract product, which has been approved and used for 10 years in China for SLIT. SLIT involves regular selfadministration at a certain time of the day; with retention of allergen extract under the tongue for 1-3 min before being swallowed. Patients are allowed to eat and drink as normal at $10 \mathrm{~min}$ after each treatment.

Dermatophagoides farinae preparation consists of five drop solutions with different concentrations of $\operatorname{Der} f$ $(1=1 \mu \mathrm{g} / \mathrm{mL}, 2=10 \mu \mathrm{g} / \mathrm{mL}, 3=100 \mu \mathrm{g} / \mathrm{mL}, 4=333 \mu \mathrm{g} / \mathrm{mL}$, and $5=1,000 \mu \mathrm{g} / \mathrm{mL}$ ) to be administered over the first five weeks of SLIT, as shown in Table 3. In patients under the age of 14 years, the daily dosing schedule for the first to third week is applied as escalating doses, and for the fourth and fifth weeks as maintenance doses. In patients aged 14 years or older, the daily dosing schedule for the first to the fourth week is applied as escalating doses, and for the fifth week as maintenance doses.

The safety profile of SLIT appears to be superior to that of subcutaneous therapy in terms of the incidence of severe SRs; however, the caveat is that such incidents typically occur away from expert care. Reported serious adverse effects such as anaphylaxis during sublingual treatment have been infrequent, with several reported events to date (190-193). In clinical trials as well as post-marketing surveys over the last 2 decades, adverse reactions have been reported in $10-15 \%$ of patients receiving SLIT and have been classified as mainly local non-life-threatening, selfremitting episodes. Most patients develop discomfort in the early phase of treatment, including oropharyngeal pruritus and angioedema.

As SLIT is self-administered, it is important to give patients and their guardians clear information about the nature and likelihood of unwanted events and simple, written instructions on the steps to take if they arise, as well as advice on the storage of sublingual vaccines securely out of the reach of children. All patients should have access to telephone advice and the opportunity to be seen at short notice.

In conclusion, more than 2 years of SLIT can acquire stable therapeutic effects. In China most doctors consider that at least 3 years of SLIT is required. It has also been shown that, when SLIT is continued perennially, clinical and immunological changes occur in successive years of treatment, although whether this requires daily as opposed to less frequent treatment is unknown.

\section{Cluster specific immunotherapy}

For conventional AIT, the duration of build-up phase depends on the frequency of the injections (normally 1 to 2 times per week, with a single injection given at each visit) and generally ranges from 3 to 6 months (194). The lengthy build-up period is one of the drawbacks of conventional AIT, which leads to low treatment compliance, especially in China, due to the uneven health service and transportation.

Cluster AIT is a variation of conventional AIT, in which the period from the build-up phase up to the therapeutic maintenance phase is much shorter than in conventional AIT (195). The protocols of cluster AIT have been investigated in several clinical trials using chemically modified allergen extracts (179,196-199). Compared to the normal build-up phase of around 4 months in conventional AIT, the build-up dosing phase in cluster AIT lasts between 6 to 8 weeks, and is followed by a dose-maintenance phase (Table 4). Cluster AIT involves 
Table 4 Comparison between cluster and conventional immunotherapy schedules

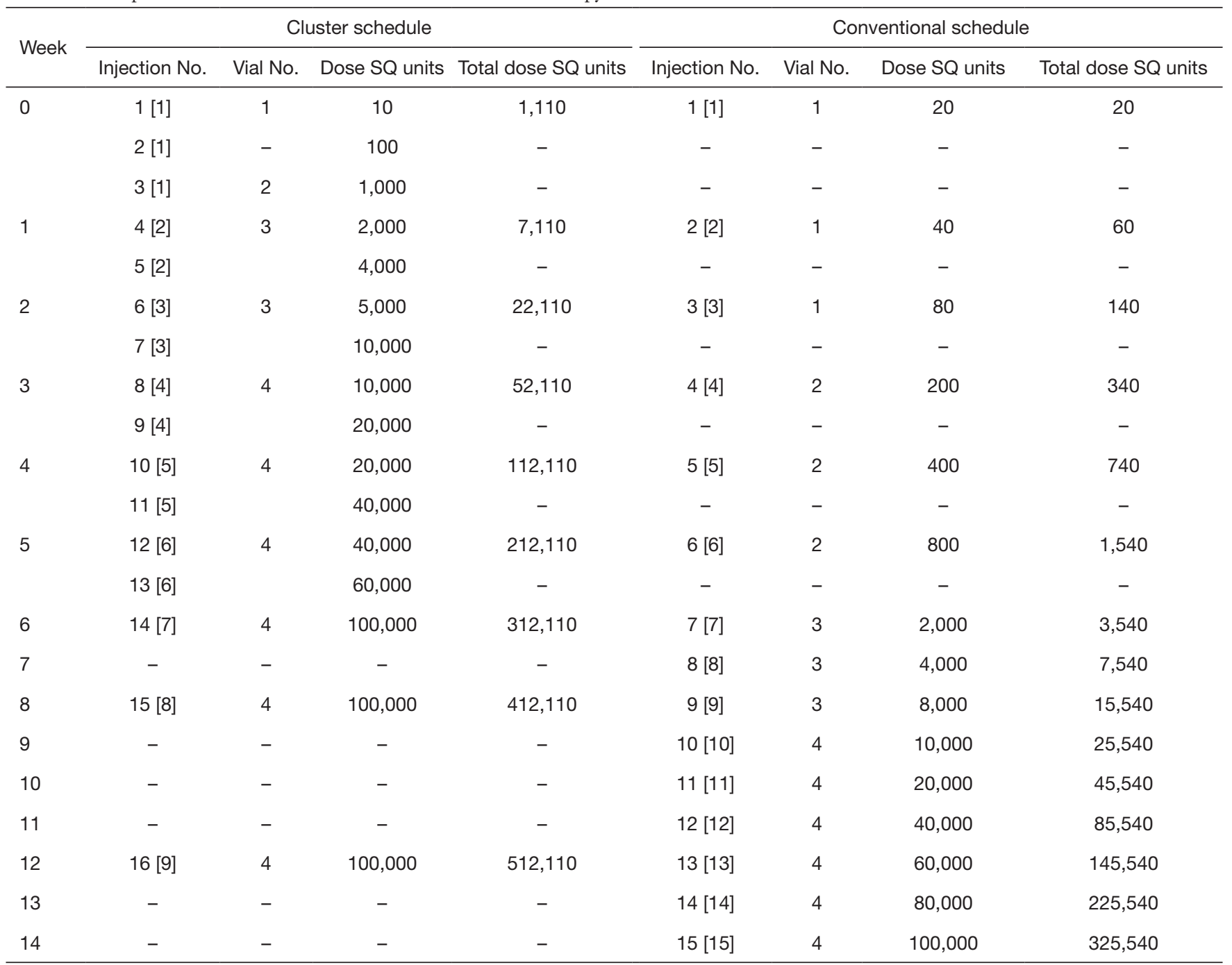

Figures in square brackets are visit No. SQ, standard quality.

administration of a commercial standardized depot extract preparation; which has usually been prepared by adsorption on aluminum hydroxide and then standardized in standard quality (SQ) units. The highest-concentration vial has an allergenic activity of $100,000 \mathrm{SQ} / \mathrm{mL}$, which is diluted accordingly for initial doses. Two injections (3 injections for the first visit) with specific concentrations of allergen are given at each visit, with a half-hour interval between injections. The patients are observed for another $30 \mathrm{~min}$ after the last injection of each cluster. The maximum dose of 100,000 SQ is administered at the 6th or 8th week, and the dosing interval is then gradually increased to 6 weeks and maintained until the end of the first year (26). The total treatment schedule is around 3 years.
All patients need to come to the clinic for treatment throughout the schedule and are pre-treated with an antihistamine (10 $\mathrm{mg}$ loratadine), at least $1 \mathrm{~h}$ before each visit. To ensure safety, PEF is recorded before and $1 \mathrm{~h}$ after the injections for each asthma patient (26). Patients are excluded from the schedule if treatment has been interrupted for longer than 5 weeks during the initial phase or longer than 16 weeks in the maintenance phase, or if severe adverse SRs occur.

Even though cluster AIT is an attractive alternative to conventional AIT, this scheme has the drawback of the risk of potentially life-threatening local or SRs $(196,200,201)$. Several studies have investigated the safety of cluster AIT with HDM or grass pollen. One meta-analysis showed that 
Table 5 Rush immunotherapy schedule

\begin{tabular}{lccccc}
\hline Build-up phase & No. of injection sequence & No. of injection bottle & Target volume $(\mathrm{mL})$ & Target dose $(\mathrm{SQ}, \mathrm{U})$ & Time of injections \\
\hline Day 1 & 1 & 1 & 0.1 & 10 & $8: 30$ \\
& 2 & 2 & 0.1 & 100 & $10: 30$ \\
\multirow{2}{*}{ Day 2 } & 3 & 3 & 0.1 & 1,000 & $14: 30$ \\
& 4 & 3 & 0.2 & 2,000 & $8: 30$ \\
& 5 & 3 & 0.4 & 4,000 & $10: 30$ \\
Day 3 & 6 & 3 & 0.6 & 6,000 & $14: 30$ \\
& 7 & 3 & 0.8 & 8,000 & $8: 30$ \\
& 8 & 4 & 0.1 & 10,000 & $10: 30$ \\
Day 4 & 9 & 4 & 0.2 & 20,000 & $14: 30$ \\
& 10 & 4 & 0.3. & 30,000 & $8: 30$ \\
Day 5 & 11 & 4 & 0.4 & 40,000 & $14: 30$ \\
Day 6 & 12 & 4 & 0.5 & 50,000 & $8: 30$ \\
\hline
\end{tabular}

$\mathrm{SQ}$, standard quality.

no differences existed in the incidence of either local adverse reaction or systemic adverse reaction between the cluster group and control group (194). Although several studies suggest that cluster AIT is a safe routine clinical practice $(195,196,202,203)$, Other studies have indicated that the adverse reactions range from $1 \%$ in patients receiving a conventional schedule, to $0-79 \%$ in patients receiving cluster SIT $(26,203)$. Thus, from this point of view, the safety of cluster AIT needs to be further investigated and confirmed in other larger trials.

\section{RIT}

The efficacy of AIT is proven and evident. As mentioned above, the drawbacks of this treatment are its inconvenience of administration and lengthy duration, which pose barriers to treatment compliance of patients living far away from hospitals or clinics. Due to time and travelling issues, many patients cannot benefit from this treatment.

In this regard, RIT requires less time for the buildup phase. Compared with conventional immunotherapy and cluster immunotherapy, the build-up phase of RIT is reduced from 15 and 7 weeks, respectively, to less than 1 week. However, there is no standard RIT protocol around the world. Generally, 1 injection every 1-3 hours or even 1 injection every 15-60 minutes over a period of maintenance phase from about 4 hours to 7 days has been employed (29,203-205). RIT is currently used mostly for bee venom and grass pollen immunotherapy. Apart from SLIT, dust mites subcutaneous RIT has been reported less frequently (29,206-208). Also, there is some evidence that the shortened treatment time of RIT, results in improved patient compliance to high allergen doses on resuming a conventional AIT schedule (209).

As a developing country, China has very diverse living standards among the population due to differences in infrastructures, transportation and health services. To save time and resources and minimize costs, RIT may be more suitable for China, considering its economic condition. As AR in most patients is caused by HDM (20), there is only RIT for standard mite antigen in China. In RIT, 1-3 injections are administered at 2 hourly intervals every day for 7 days, by which time the maintenance phase has been reached (Table 5). The schedule at maintenance phase is then the same as for the conventional treatment schedule. To ensure safety, patients in RIT build-up phase need to be hospitalized for six days of treatment, and are given oral antihistamines and glucocorticoids. The medication 
for adults includes loratadine $10 \mathrm{mg}$ /day and prednisolone $8 \mathrm{mg} /$ day, with half this dose for children. In order to detect patients with asthma, lung function tests should be performed. Before injection, PEF is recorded, recording the highest value among three readings. If the average PEF predictive value is $80 \%$ or less, the patient can be started on RIT and injected under close observation. If the average PEF predictive value is $70 \%$ or less, the RIT injection should be delayed until PEF returns to normal after treatment with medication. One recent study by Xie and colleagues (210) has demonstrated that in AR patients pretreated with medication (corticosteroids and antihistamines), the safety and efficacy of RIT is similar to conventional immunotherapy using standard HDM allergen vaccine.

However, this kind of treatment should only be carried out by hospitals or clinics with considerable experience in immunotherapy, to avoid and deal with SRs.

\section{Patient administration and follow-up care}

\section{AR patient}

\section{Patients administration}

$\mathrm{AR}$ is a chronic disease, but patients should be encouraged that onset and development of the disease could be controlled through avoidance of allergen exposure and acceptance of appropriate treatment (211).

Patients are encouraged to establish partnership with medical staff to formulate a long-term control and management plan, which should be adhered to. An individualized treatment scheme is developed depending on the work and life situation of the patient, and a thorough understanding of the features of common allergens. Medical staff should help patients to understand the nature of AR, be familiar with the occurrence of an anaphylactic shock and corresponding treatment, and correctly grasp the medication techniques to reduce different kinds of the adverse reactions. Cooperation between medical staff and patients will reduce medical visits and improve compliance during AIT treatment (211).

AIT in patients with AR should start as early as possible. The effectiveness of AIT should be informed comprehensively and appropriate; including symptom improvement, reduction of medication dosages, prevention of the progression from AR to asthma and the development from monosensitization to polysensitization, induction of tolerance to allergens, and achieving the goal of long-term remission $(47,212)$.

The limitations of specific immunotherapy should also be clarified to the patient. Patients should be aware that allergen avoidance and symptomatic treatment cannot be ignored during immunotherapy, and also be informed about the possible adverse reactions, duration of treatment and approximate costs, before signed informed consent is obtained. When treatment is to be given to a very young child, the long-term treatment plan should be formulated and the signed informed consent should be obtained from the child's legal guardian $(46,213)$.

\section{Follow-up care}

Patients receiving AIT should be followed-up strictly and reviewed to assess both the disease control and the local and SRs after injection. The assessments include symptom and medication scores, correctness of medication method, delayed adverse reaction/s, annual review of allergen SPT and changes in levels of sIgE and IgG4. Nasal fractional exhaled nitric oxide (FeNO), and cell counts in nasal secretion smear, especially the number and proportion of eosinophils, can be monitored in medical institutions $(46,214)$.

\section{AR with asthma}

$\mathrm{AR}$ and asthma commonly coexist due to their similarities in anatomy, physiology and immunopathology (1). The incidence of AR among asthmatic children is nearly $50 \%$ in China and 60-80\% worldwide (215-217). AR impacts asthma morbidity and is associated with poorer asthma control as well as impairment of daily life activity. Thus, treatment of AR may be beneficial in improving asthma control $(218,219)$, which may also support the hypothesis of "one airway, one disease" (220).

Objective measurements, such as PEF (221) and FeNO (222), are effective parameters when accessing disease severity or adjusting anti-inflammatory interventions in patients with AR and asthma. Subjective measurements, including questionnaires on nasal and ocular symptoms, asthma control and health-related quality of life (HRQoL) can also be used to evaluate the efficacy of AIT (223).

In order to provide standard options to facilitate asthma management in China, Chinese Pediatric Asthma Group have aimed to establish the national pediatric asthma collaborative network among more than 2,000 hospitals and medical centers and to share a national network with unified asthma diagnosis and treatment strategies. It is envisaged that utilization of electronic monitoring devices 
and internet-based apps may help to complete the network interactivity; particularly as some studies have shown that asthma self-management through electronic devices or internet-based apps can provide higher adherence, better monitoring in asthma symptoms, medical usage and asthma exacerbations $(224,225)$. Thus, similar management methods could be used for AR patients, and patients with both diseases may benefit from the selfmanagement by promoting disease control, awareness of symptoms, treatment adherence, and parent-physician partnerships.

\section{Pharmacotherapy before, during and after immunotherapy}

In the management of AR, co-administration of AIT and pharmacotherapy can increase the clinical effect and safety of treatment. Medicines before, during and after immunotherapy should be administered according to an individual's requirements (125).

\section{Drug use before immunotherapy}

During the onset of AR, severe symptoms that dramatically deteriorate quality of life urge patients towards an instant symptom-relieving therapy rather than an immunotherapy that functions effectively but slowly. In this case, antiallergic drugs are preferable, such as oral $\mathrm{H1}$-antihistamines and/or nasal corticosteroids. These drugs can effectively control the symptoms and lay a foundation for further immunotherapy.

\section{Drug use during immunotherapy}

Drugs are used during immunotherapy to lessen symptom rebounds and adverse reactions. Based on the severity/ seriousness of potential adverse reactions, appropriate drug administration should be accomplished to prevent the development of such adverse reactions and their subsequent damage to the body (28).

The adverse reactions of immunotherapy can trigger severe asthma that is life-threatening. Therefore, for patients with AR and comorbid asthma, AIT is recommended in combination with drugs such as inhaled corticosteroids, bronchodilators, and leukotriene receptor antagonists. In this way, patients' tolerance against allergens can be enhanced, asthma relieved, and quality of life improved.

\section{Drug use after immunotherapy}

Although AIT has a long-term effect, relapse of AR occurs in some patients. Appropriate pharmaceutical intervention, based on detection and control of inducing factors, can be chosen to alleviate the symptoms and consolidate therapeutic outcomes in these patients.

\section{Measures of efficacy}

\section{Rbinitis control}

There are many ways to evaluate the efficacy of immunotherapy, and these can be categorized according the patient's own assessment and the doctor's assessment $(226,227)$. The World Allergy Organization (WAO) has recommended that for assessment of the efficacy of AIT, the primary endpoint parameters of the evaluation should be the severity of the symptoms and use of concomitant medications, and these two primary endpoints should be considered together in the assessment. Furthermore, the secondary endpoint parameter should be the degree of improvement of patient's quality of life.

The main goals of evaluating the efficacy of immunotherapy are as follows $(1,228)$ : (I) to record the patient's symptoms and their response to immunotherapy, to provide the basis for therapeutic dosage adjustment in future; (II) to provide data to continue treatment; (III) to provide data for research; and (IV) to assess the overall effectiveness of immunotherapy.

The evaluation of clinical efficacy of immunotherapy includes measurement of nasal symptoms score, quality of life score, and the drug dosage score.

\section{Nasal symptoms sore}

Assessment of specific nasal symptoms, including "nasal congestion", "sneezing", "nasal itching", and "nasal secretion" is necessary in the evaluation of AR (1). Each symptom is scored between 0 and 3 , so that the total nasal symptoms score ranges from 0 to 12 . A similar scoring system can be used for ocular symptoms, including "eye itching", "ocular secretion" and "eye redness", to attain a total ocular symptom score that ranges from 0 to 9 . Nasal symptoms and medication can be evaluated daily using a computer software-based documentation system, whereas quality of life can be assessed each year (229). VAS is generally recommended for subjective symptom evaluation by patients $(1,228)$. The VAS can be a good means for assessing the patient's feelings about the severity of their 
disease, especially during long-term follow-up of AR.

\section{Quality of life score}

To assess the overall efficacy of AIT, not only nasal symptoms, but also the patient's mental/psychological condition, and the HRQoL need to be evaluated (230). In this regard, the RQLQ is the prevalent scoring system employed (231).

\section{Medication score}

Although AIT treatment can relieve the symptoms of AR, few patients are completely symptom-free (1). Clinicians may give medications to relieve symptoms as appropriate, but it is particularly important to record concomitant medications during the process. According to WAO guidelines, the nasal, ophthalmic and oral antihistamines used during AIT should be rated as 1 point, the inhaled or nasal corticosteroids as 2 points, and oral corticosteroids as 3 points. The overall "symptom load" is expressed as the sum of the symptom score and the medication score.

Subjective evaluation of the degree of improvement in symptoms may also use the following criteria (232): (I) no efficacy, less than $30 \%$ improvement; (II) slight efficacy, $30-45 \%$ improvement; (III) moderate efficacy, 45-60\% improvement; (IV) significant efficacy, more than $60 \%$ improvement.

\section{Asthma control}

The efficacy of AIT in asthma is measured using symptom scores, medication scores, as well as lung function test (55,134,233-235). The symptom and medication scores are frequently used as primary endpoints in AIT trials. The daytime asthma symptoms are scored from 0 to 4 or 5 points according to the general severity of wheeze, shortness of breath, dyspnea, cough and its impact on daily life. The nocturnal asthma symptoms are scored from 0 to 4 points according to the frequency of nocturnal and early morning awakening by asthma. The total asthma symptom score (TASS) is the sum of daytime and nocturnal asthma symptoms scores, and generally been evaluated monthly in most studies $(54,55,134,234,235)$.

With regard to the medication allowed in the AIT studies, antihistamines, antileukotrienes or $\beta 2$-agonists, topical corticosteroids as well as oral corticosteroids were evaluated by equivalent scores. All these studies demonstrated that the AIT treated group had a significant decline in the medication score compared with the placebo group $(54,55,134,234)$.

Some studies have evaluated asthma control ("well controlled" or "totally controlled") and asthma symptom score according to GINA guideline as well as the number of emergency room visits or hospitalisation for asthma attack $(234,236,237)$. In one of the studies, a subsequent post hoc analysis by asthma severity revealed significant clinical benefit in the AIT group with moderate, persistent asthma at baseline with 401-800 $\mu \mathrm{g}$ budesonide/day; with greater achievement of well-control asthma and totally controlled asthma (236). In another study, global assessment of asthmatic symptom by patient and physician, the primary outcome measure, was improved after ASIT but not placebo treatment (233).

Most studies that included the measurement of lung function parameters showed significant improvement before and after AIT. The parameters included $\mathrm{FEV}_{1} \%$ predicted, $\mathrm{PEF}$ rate in the morning and night, $\mathrm{PEF} \%$ predicted value as well as brochoprovocation tests $(54,55,233-235,237)$. However, within-group analysis in some studies failed to demonstrate significant differences between AIT and control groups $(233,234,236)$.

\section{Biomarkers}

Both SCIT and SLIT are characterized by the induction of Treg and B cells, decreased allergen-specific T-cell proliferation, a shift from TH2 to TH1 cytokines, and a shift from an IgE to an IgG4/IgA antibody response (238). Moreover, there are several aberrant changes in the periphery or target organs during the treatment, which can be regarded as putative markers of successful immunotherapy, or for the consideration of whether or not to stop the treatment. A good biomarker should be specific, easy to examine, and has prediction or correlation with the clinical response of the treatment.

\section{IgE-facilitated allergen binding (IgE-FAB)}

$\mathrm{IgE}-\mathrm{FAB}$ is an important method to test the active inhibitory properties of antibodies after immunotherapy. The allergen- $\operatorname{IgE}$ complex, formed by the incubation of allergen and high-concentration sIgE, can bind to the low-affinity IgE receptor expressed on EBV-B cells and be detected by flow cytometry (239). Shamji and colleagues (240) examined $\mathrm{IgE}-\mathrm{FAB}$ after grass pollen immunotherapy and found that the immunoreactive levels of IgG4 correlated closely with the clinical response to grass pollen immunotherapy. Another study has also shown that the immunoreactive levels 
of IgG4, different from the concentration of specific IgG4, were maintained unchanged after 2 years of discontinuation of immunotherapy (241); suggesting that the examination of immunoreactive levels of IgG4, rather than direct measurement of the concentration of IgG4 or other antibodies, is likely to be a superior test of the efficacy of immunotherapy. However, the preparation of EBV-B and demand of flow cytometry may limit the use of the method.

\section{IgE blocking factor}

IgE blocking factor is also a biomarker to test for the presence of bioactive antibodies which can block the binding of sIgE and allergens after immunotherapy. Good correlation has been found between $\mathrm{IgE}-\mathrm{FAB}$ and $\mathrm{IgE}$ blocking factor. The difference between IgE blocking factor and $\mathrm{IgE-FAB}$, however, is that in the assessment of $\mathrm{IgE}$ blocking factor, antibodies are used to compete with sIgE when binding the allergen on a solid-phase matrix. In contrast, IgE-FAB represents a more physiologic readout of functional IgG1 and IgG4 that seems to correlate more closely with symptom and medication scores (241).

\section{Ratio of sIgE to total IgE (tIgE)}

During the immunotherapy, sIgE increases transiently at the beginning of treatment, followed by a gradual decrease over time. The change of serum $\mathrm{sIgE}$ is limited to reflect or predict the immune or clinical efficacy of immunotherapy (242).

The ratio of serum $\mathrm{sIgE}$ to $\mathrm{tIgE}$ has recently been studied as a potential predictive marker of immunotherapy (243). In patients who received 4 years of SCIT or SLIT, there was a significant correlation between the serum $\mathrm{sIgE} / \mathrm{tIgE}$ ratio and the clinical response to ASI, and that a diagnostic serum $\mathrm{sIgE} / \mathrm{tgE}$ ratio of 16.2 was found to be the cut-off point beyond which successful immunotherapy outcome could be predicted, with $97.2 \%$ clinical sensitivity and $88.1 \%$ clinical specificity. Furthermore, measurement of serum sIgE/tIgE ratio was more useful than either $\mathrm{sIgE}$ or $\mathrm{tIgE}$ individually in predicting treatment efficacy in patients mono-sensitized to grass, Parietaria judaica, Olea europea, and HDM (243). As not many studies have reported the use of $\mathrm{sIgE} / \mathrm{tIgE}$ ratio as a biomarker for effective immunotherapy, this needs to be confirmed in more randomized double-blind placebo-controlled studies to confirm its suitability in this regard.

\section{Specific IgG (IgG4)}

Specific IgG subclasses have been fully studied during immunotherapy (244). Allergen specific IgG1 and IgG4 concentrations in serum increase after immunotherapy for several weeks, while allergen specific IgG2 and IgG3 do not change significantly (245). Although the elevated concentrations of allergen specific IgG (in particular IgG4) have the potential to compete with sIgE, many studies have failed to show the correlation between increased concentrations of specific IgG1 or IgG4 and clinical efficacy of immunotherapy. The vaccines used currently for immunotherapy are usually extracts from the allergens, which may comprise multiple components. The "specific" IgG or IgG4 detected and determined after immunotherapy are actually "total" IgG or IgG4 against the multiple components in the allergens. Some antibody responses of un-related components or even contaminant antigens may interfere with the actual results, and thus, this is one reason why the increased concentration of specific IgG or IgG4 may not be related with the clinical efficacy (244).

While immunotherapy response without an increase in specific IgG4 is a sign of definite treatment failure, the immunotherapy response with an increase in specific IgG4 does not necessarily mean a consequent positive clinical response (246).

\section{Specific IgA}

Allergen specific IgA, in particular IgA2, has been reported to be increased after immunotherapy. The increase of specific IgA follows IL-10 production of Treg cells (247). As IgA is unable to mediate, complement, or participate in an immune complex formation, it is possible that this may act as an inhibitory antibody such as IgG4 (248). However, very few studies have investigated the correlation between the increase in allergen specific IgA and the clinical response to immunotherapy, and thus no conclusion can presently be made on the role of IgA in immunotherapy.

\section{Other biomarkers}

Other potential biomarkers include allergen-specific IL10-secreting $\operatorname{Tr} 1$ or $\mathrm{Br} 1$ cells; increase concentrations of cytokines IL-10 and TGF- $\beta$; decreased levels of cytokines IL-4 and IL-5; and decreased inflammatory cells such as eosinophil and mast cells (238). These biomarkers are present in either very low quantities to be examined (allergen-specific IL-10-secreting Tr1 or Br1 cells) or are non-specific (the cytokines or inflammatory cells), and therefore not suitable as 
Table 6 Conditions potentially associated with subcutaneous immunotherapy-related systemic reactions

\begin{tabular}{l}
\hline Risk factors \\
\hline Administration-related factors \\
Missed dose adjustment during pollen peak \\
Allergen composition and mixtures \\
Accelerated build-up regimens \\
Short time of observation after injection \\
Needle length $\geq 13$ mm \\
Delayed epinephrine administration \\
Dosing errors \\
Non-specific patient-related factors \\
Uncontrolled asthma (contraindication) \\
Long-term therapy with non-cardio-selective b-blockers and \\
ACE inhibitors \\
Specific patient-related factors \\
Previous systemic and local reactions \\
High degree of allergen sensitivity
\end{tabular}

ACE, angiotensin converting enzyme.

biomarkers for immunotherapy.

\section{Safety of immunotherapy}

\section{Risk factors of adverse reactions}

AIT is contraindicated in patients with medical conditions that might increase the risk of treatment-related severe SRs, such as those with severe or poorly controlled asthma or with significant cardiovascular diseases (e.g., unstable angina, recent myocardial infarction, significant arrhythmia, and uncontrolled hypertension). Thus, AIT should be administered with caution to patients receiving $\beta$-blockers or angiotensin-converting enzyme inhibitors. Risk factors for SCIT-related SRs include symptomatic asthma, prior SCIT-related SRs, and a high degree of skin test reactivity (27). Other potential risk factors for SCIT-related SRs, such as administration during the height of the pollen season, build-up dosing schedule (cluster vs. conventional), and treatment phase (maintenance $v s$. build-up phase) have been suggested, but none have been clearly established (249). However, some risk factors for severe adverse effects and fatal events are shown in Table 6 (250-253).

\section{Adverse reactions}

\section{LRs}

LRs in AIT refer to any symptoms or signs located at or nearby the area of administration. These are not rare, and the common manifestations of SCIT are pruritus, redness, and/or swellings except pain that everybody endures at the time of injection. These usually appear within 30 minutes after injection, sometimes after several hours or after one day. The larger LRs (LLRs) are defined as the maximum diameter greater than $2.5 \mathrm{~cm}$ before, now bigger than the patient's palm (average adult, $8 \mathrm{~cm}$ ). The frequency of LRs, however, ranges from $26 \%$ to $82 \%$ of patients and with $0.7 \%$ to $4 \%$ of injections used (254-256). One 3 -year follow-up retrospective study found that although the incidence of SRs did not increase significantly in patients with the LLRs (257), some individuals with higher frequencies of LLRs were at greater risk of developing SRs $(258,259)$. One retrospective study indicated that LRs following an injection cannot predict occurrence of LRs at the next injection (27). The LRs of SLIT include oral and gastrointestinal reactions such as lip swelling, oral mucosal pruritus, burning, uvula edema, abdominal pain, vomiting and diarrhea. Oral mucosal reactions, are relatively common, resolve within a few days or weeks without medical intervention, and affect up to $75 \%$ of patients, most usually in the build-up phase (118).

\section{SRs}

Based on the literature, the incidence rate of adverse systemic reaction in AR ranges from 2.6\% to 14\% (260-263). The incidence rate of adverse systemic reaction per injection is about $0.1 \%$ to $0.2 \%(249,264)$, while the incidence of fatal severe reaction is one event per 1 to 2.5 million injections (265-267). The risk factors associated with the systemic reaction include uncontrolled asthma, dosing error, high sensitivity of the skin, administration of $\beta$-receptor agonist, history of SRs associated with SCIT, administration of injections during allergy season, insufficient observation time after injection, injection operated in the clinic without the surveillance by clinicians, and delayed administration of epinephrine. Among the above factors, uncontrolled asthma is the most important risk factor; therefore, controlling the asthmatic symptoms is critical to prevent the severe SRs. Compared to SCIT, SRs rarely occur in SLIT. The incidence rate of systemic reaction in SLIT is about $0.056 \%$ per administration and no death has been reported (268). 
The classification of SRs of SCIT commonly used in mainland China follows that proposed by the European Academy of Allergy and Clinical Immunology (EAACI) Immunotherapy Task Force guideline in 2006 (74). However, the consensus amongst specialists in China is that the classification system proposed by WAO in 2010 (269) is clearer and more detailed. The WAO grading system is considered to be more practical and convenient for the clinicians to make the diagnosis. The EAACI and WAO classification systems for SRs of SCIT are shown as Table 7.

Notes for WAO classification system:

(I) Severe LRs with SCIT may rapidly develop into SRs (270);

(II) The cutaneous presentations in grade I SRs may rapidly progress into more severe reaction;

(III) Patients may have a feeling of impending death, especially in grades III or IV reactions; but children with severe reactions may have some changes in their behavior, e.g., becoming very quiet or irritable and cranky;

(IV) The most common treatment for SRs is subcutaneous injection of 0.3 to $0.5 \mathrm{~mL} 1: 1,000$ epinephrine, especially for grades III and IV SRs. If symptoms do not relieve, use epinephrine again 15-20 minutes later if necessary;

(V) It is suggested to stop SCIT if grade III or IV SRs happened.

\section{Management of adverse reactions}

Generally, most of the patients are tolerant to AIT, but adverse reactions occurring in some patients during the procedure require treatment accordingly. This is common practice for LRs in both SLIT and SCIT, but especially in SLIT. For mild LRs, these can usually be relieved by local application of cold compresses or glucocorticoid cream, or by decreasing the dosage of the vaccine, with or without oral antihistamine (271). Nevertheless, patients with mild adverse reaction should be observed continuously and any worsening of symptoms should be evaluated carefully as potential signs of anaphylaxis.

For SLIT, it has to be emphasised that the patients should understand the possible adverse reaction/s they might experience during the procedure and know how to act when taking the vaccine at home; even though no severe systemic adverse reactions have occurred to date in China.

For local severe adverse reactions and SRs, more attention should be paid because of both their severity and potential to sometimes result in anaphylaxis, especially for SCIT $(259,272)$. Early signs should be identified, including palms and soles itch, perianal or peri-genital pruritus, bellyache and an urge to defecate and urinate, sneezing attacks and generalized pruritus. Subsequently, respiratory and/or cardiovascular symptoms may appear (273). The management of severe local adverse reactions and systemic adverse reactions in SCIT is detailed in Table 8 (31,272-274).

As adverse reactions usually occur within $30 \mathrm{~min}$ after the injection, especially for SCIT, the patient must be supervised for at least $30 \mathrm{~min}$ following injection. Also, injections must be given by clinicians in the hospital, where there is a well-equipped emergency facility with the following apparatus: (I) trolley for patient to lie flat if needed; (II) electrocardiograph, pulse oximetry and blood pressure monitoring equipment; (III) oxygen and suction equipment, including tubing, masks, etc.; (IV) airway management equipment and advanced airway management devices for intubation and cricothyrotomy; (V) an intravenous infusion pump; (VI) nebulizer mask (for inhaled epinephrine); (VII) manual blood pressure cuff; (VIII) intravenous access cannulae (20-16 G) and giving sets; (IX) needles and syringes $(74,275)$.

As recommended in guidelines, it is essential to carefully examine the patient and take medical history before administration of every injection. PEF evaluation must be done before each injection, especially for the patients who have comorbid asthma with AR. PEF value of less than $70 \%$ of best predicted is considered as a warning signal (74). Most severe systemic adverse events would be prevented if physicians abide by these rules.

\section{Safety comparison}

When administered correctly to properly selected patients in a hospital with experience, both SCIT and SLIT are safe and well-tolerated $(118,276,277)$. Generally, SLIT is safer than SCIT (192). Patients treated with SCIT are at risk of both local and systemic adverse reactions, which may be severe, even life-threatening. Occasionally, system allergic reactions occur during SLIT treatment, but life-threatening anaphylaxis is extremely rare. About $0.1 \%$ to $3.5 \%$ of injections have been shown to result in a systemic allergic reaction for SCIT, compared to a $0.056 \%$ rate of systemic allergic reactions per SLIT dose. Fatal cases have been reported with SCIT, but not SLIT (249).

Adverse reactions have been shown to occur in 


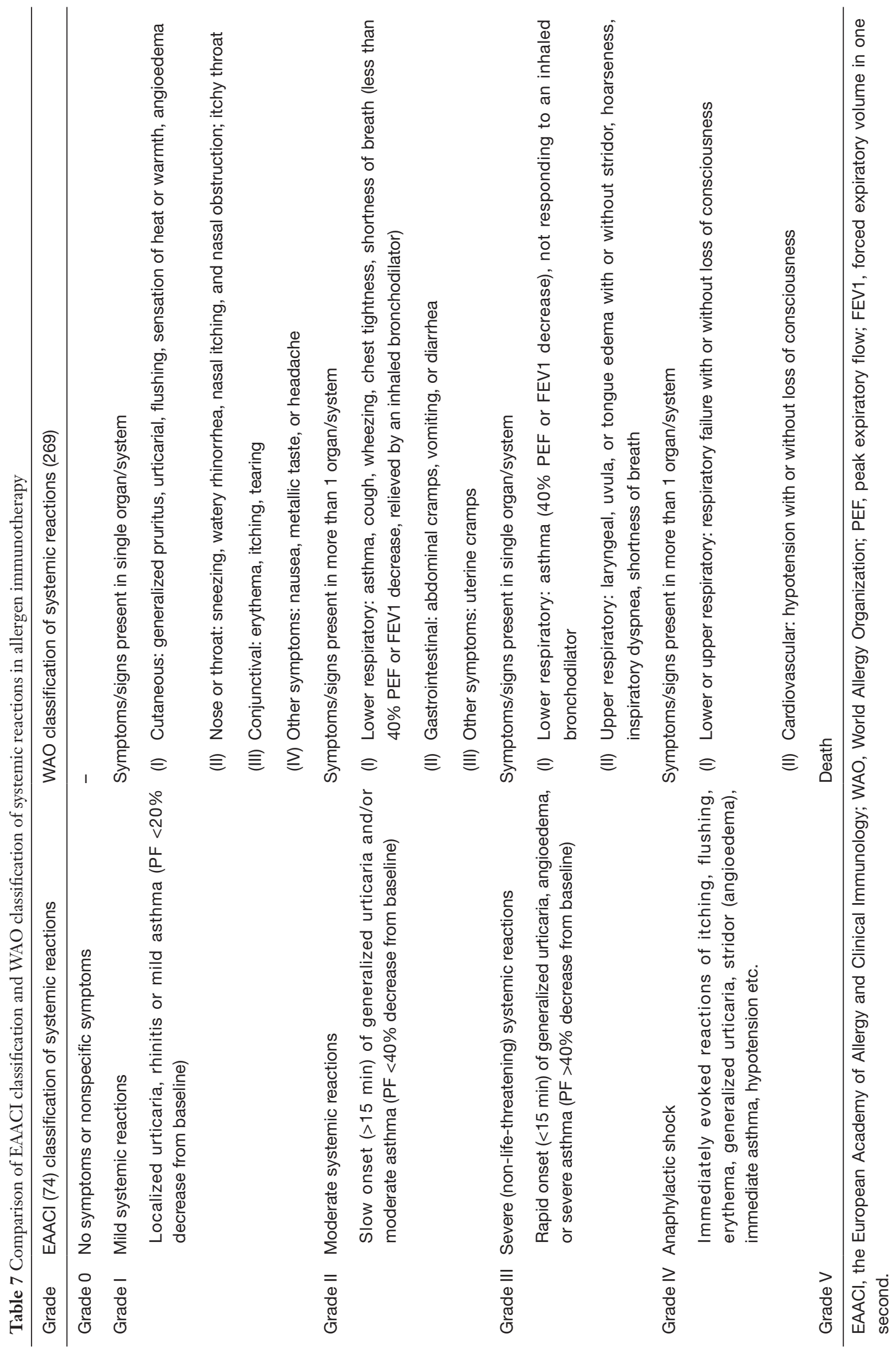




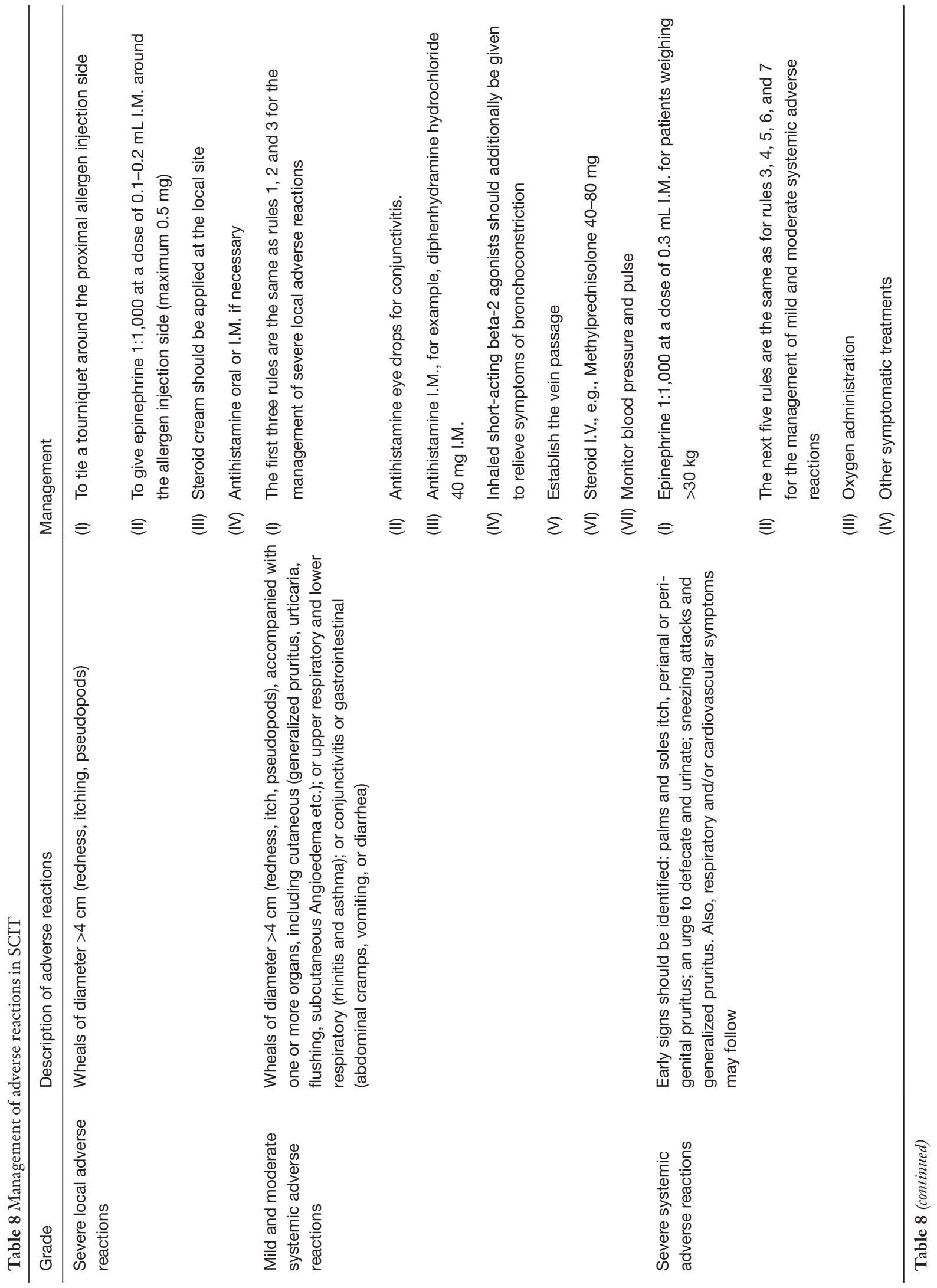




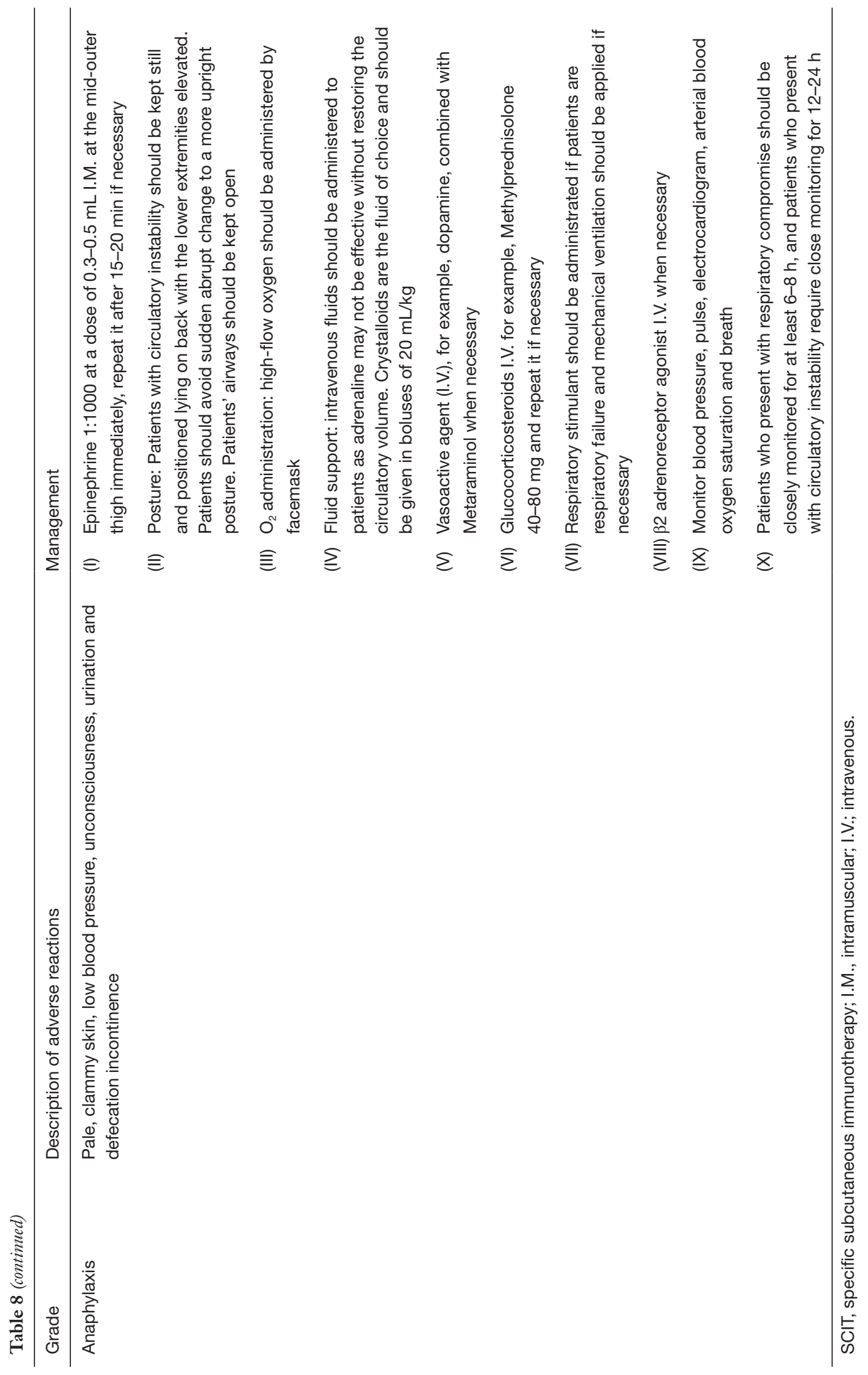


Table 9 Grading system for local adverse events in sublingual immunotherapy (SLIT) according to the World Allergy Organisation (279)

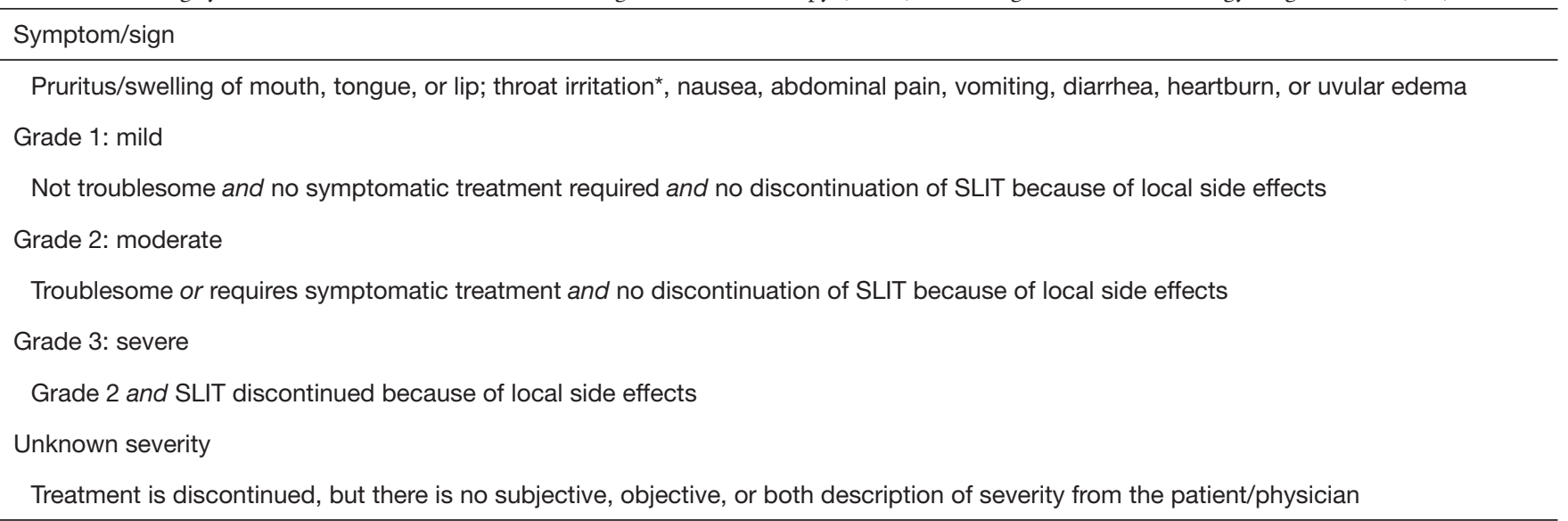

Each local adverse event can be early $(<30 \mathrm{~min})$ or delayed. * , for example, itchy palate, burning or swelling of the throat.

$10 \%$ to $20 \%$ of patients who were prescribed SLIT $(54,134,271,277,278)$. However, manifestations are temporary mild local mucosal reactions in $40 \%$ to $75 \%$ of the cases, and usually occur during the early phase of SLIT, before gradually subsiding after 1 to 3 weeks (54). The other adverse reactions due to SLIT include nausea and/or abdominal pain (particularly in children), rhinitis, conjunctivitis, headache, urticaria, cough and bronchospasm (192). Recently, the WAO has proposed a new, standardized classification of LRs during SLIT to provide a worldwide standardized reporting system (Table 9) (279).

Although there is no report of severe anaphylaxis due to SLIT in China (280), some cases with severe anaphylaxis have, nevertheless, been reported in other countries $(278,281)$. Normally, SLIT is self-administered at home without the supervision of physicians and nurses. However, guidelines for some SLIT products indicate that the first one or more doses should be administered under the supervision of physicians and nurses in hospital, and thereafter self-administered at home. The patients should be informed of the situations in which SLIT should be temporarily interrupted; including elective maxillofacial surgery, oropharyngeal infections and lesions (ulcers, gingivitis, periodontitis), gastroenteritis and asthma exacerbations (118). All patients should be required to have access to telephone advice and opportunity to be seen at short notice during administration of SLIT.

Generally, SLIT has a better safety profile than SCIT. SRs are far less often compared with SCIT, and lifethreatening anaphylaxis is extremely rare.

\section{Summary and future perspective}

AIT achieves substantial clinical results in patients with AR by improving nasal and ocular symptoms, reducing medication need, improving quality of life, preventing progression of AR to asthma, and reducing new sensitizations. The efficacy of immunotherapy depends on correct patient selection, the type of allergen and the product chosen for treatment. Each vaccine requires individual assessment through a combination of clinical history and skin and/or blood tests for allergen $\operatorname{sgE}$ before recommendation for routine use.

Patients receiving immunotherapy should be carefully monitored for at least 30 minutes after administration of therapy and SRs treated promptly. Both SLIT and SCIT have acceptable safety profiles if administered under the appropriate circumstances. A superior safety profile of SLIT allows for administration outside of a medically supervised setting, whereas SCIT is recommended only in a medically supervised setting with appropriate staff and equipment to identify and immediately treat anaphylaxis. Cluster immunotherapy and RIT could overcome the inconvenience of conventional immunotherapy by shortening the build-up phase and achieve obvious efficacy. However, the potential of adverse reactions, especially SRs, increasing during the short build-up phase is real. Therefore, these two schedules should be performed under close observation of professionals with considerable experience in immunotherapy. Figure 4 shows the steps for assessment of patients suitable for immunotherapy and administration of specific immunotherapy. 


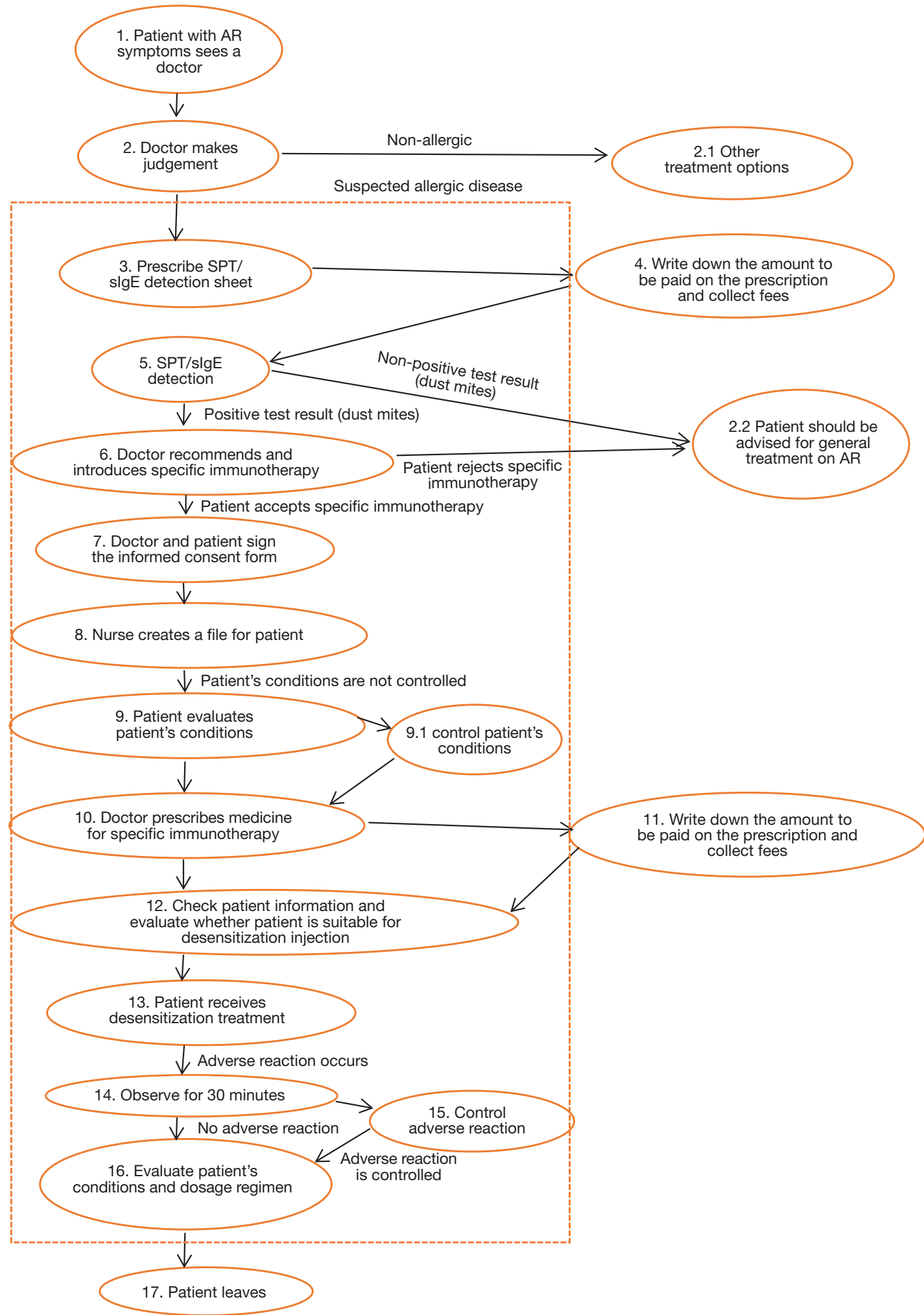

Figure 4 Flow chart for administration of specific immunotherapy. The scope surrounded by red dot lines is the scope for the institute. AR, allergic rhinitis; SPT, skin prick test; sIgE, specific immunoglobulin E. 
Future studies investigating the efficacy and safety of AIT in China need to be developed in the following areas:

(I) Standardization, validation and general acceptance of allergen products (the major allergen content);

(II) Identification of biomarkers to select responders, echo responsiveness to immunotherapy and indicate when relapse is imminent;

(III) Multicenter studies to evaluate the risk and benefit of AIT in younger children and determine optimal age for initiation;

(IV) Optimization of AIT schedules and dosing which balance both efficacy and safety;

(V) Pharmacotherapy before and during AIT to improve safety profile;

(VI) Production of auto-adrenaline injector for patients in case of SRs occurring out of a medically supervised setting;

(VII) Cost-effectiveness analysis adjusted to socioeconomic differences within China;

(VIII) Head-to-head trials comparing efficacy of SCIT with SLIT.

\section{Acknowledgements}

Funding: This work was supported by grants to L Zhang from National Key R \& D Program of China (2016YFC20160905200), the Program for Changjiang Scholars and Innovative Research Team (IRT13082), the National Natural Science Fund for the Major International Joint Research Program (81420108009), the National Natural Science Fund (81630023, 81100704, 81400444, 81441029, 81441031 and 81570894), and Beijing Municipal Administration of Hospitals' Mission Plan (SML20150203).

\section{Footnote}

Conflicts of Interest: The authors have no conflicts of interest to declare.

\section{References}

1. Bousquet J, Khaltaev N, Cruz AA, et al. Allergic Rhinitis and its Impact on Asthma (ARIA) 2008 update (in collaboration with the World Health Organization, GA(2) LEN and AllerGen). Allergy 2008;63 Suppl 86:8-160.

2. Grossman J. One airway, one disease. Chest 1997;111:11S-6S.

3. Spergel JM. Atopic march: link to upper airways. Curr
Opin Allergy Clin Immunol 2005;5:17-21.

4. Bell IR, Jasnoski ML, Kagan J, et al. Depression and allergies: survey of a nonclinical population. Psychother Psychosom 1991;55:24-31.

5. Cuffel B, Wamboldt M, Borish L, et al. Economic consequences of comorbid depression, anxiety, and allergic rhinitis. Psychosomatics 1999;40:491-6.

6. Timonen M, Jokelainen J, Hakko H, et al. Atopy and depression: results from the Northern Finland 1966 Birth Cohort Study. Mol Psychiatry 2003;8:738-44.

7. Postolache TT, Komarow H, Tonelli LH. Allergy: a risk factor for suicide? Curr Treat Options Neurol 2008;10:363-76.

8. Canonica GW, Bousquet J, Mullol J, et al. A survey of the burden of allergic rhinitis in Europe. Allergy 2007;62 Suppl 85:17-25.

9. Schatz M. A survey of the burden of allergic rhinitis in the USA. Allergy 2007;62 Suppl 85:9-16.

10. Schoenwetter WF, Dupclay L Jr, Appajosyula S, et al. Economic impact and quality-of-life burden of allergic rhinitis. Curr Med Res Opin 2004;20:305-17.

11. Nathan RA. The burden of allergic rhinitis. Allergy Asthma Proc 2007;28:3-9.

12. Bauchau V, Durham SR. Prevalence and rate of diagnosis of allergic rhinitis in Europe. Eur Respir J 2004;24:758-64.

13. Bachert $\mathrm{C}$, van Cauwenberge $\mathrm{P}$, Olbrecht J, et al. Prevalence, classification and perception of allergic and nonallergic rhinitis in Belgium. Allergy 2006;61:693-8.

14. Nathan RA, Meltzer EO, Derebery J, et al. The prevalence of nasal symptoms attributed to allergies in the United States: findings from the burden of rhinitis in an America survey. Allergy Asthma Proc 2008;29:600-8.

15. Katelaris CH, Lee BW, Potter PC, et al. Prevalence and diversity of allergic rhinitis in regions of the world beyond Europe and North America. Clin Exp Allergy 2012;42:186-207.

16. Zhang $Y$, Zhang L. Prevalence of allergic rhinitis in china. Allergy Asthma Immunol Res 2014;6:105-13.

17. Zhang L, Han D, Huang D, et al. Prevalence of selfreported allergic rhinitis in eleven major cities in china. Int Arch Allergy Immunol 2009;149:47-57.

18. Li F, Zhou Y, Li S, et al. Prevalence and risk factors of childhood allergic diseases in eight metropolitan cities in China: a multicenter study. BMC Public Health 2011;11:437.

19. Wang XD, Zheng M, Lou HF, et al. An increased prevalence of self-reported allergic rhinitis in major Chinese cities from 2005 to 2011. Allergy 2016;71:1170-80. 
20. Li J, Sun B, Huang Y, et al. A multicentre study assessing the prevalence of sensitizations in patients with asthma and/or rhinitis in China. Allergy 2009;64:1083-92.

21. Akkoc T, Akdis M, Akdis CA. Update in the mechanisms of allergen-specific immunotheraphy. Allergy Asthma Immunol Res 2011;3:11-20.

22. Noon L. Prophylactic Inoculation against Hay Fever. Lancet 1911;177:1572-3.

23. Durham SR, Emminger W, Kapp A, et al. Long-term clinical efficacy in grass pollen-induced rhinoconjunctivitis after treatment with SQ-standardized grass allergy immunotherapy tablet. J Allergy Clin Immunol 2010;125:131-8.e1-7.

24. Jacobsen L, Niggemann B, Dreborg S, et al. Specific immunotherapy has long-term preventive effect of seasonal and perennial asthma: 10-year follow-up on the PAT study. Allergy 2007;62:943-8.

25. Pajno GB, Barberio G, De Luca F, et al. Prevention of new sensitizations in asthmatic children monosensitized to house dust mite by specific immunotherapy. A six-year follow-up study. Clin Exp Allergy 2001;31:1392-7.

26. Zhang L, Wang C, Han D, et al. Comparative study of cluster and conventional immunotherapy schedules with dermatophagoides pteronyssinus in the treatment of persistent allergic rhinitis. Int Arch Allergy Immunol 2009;148:161-9.

27. Cox L, Nelson H, Lockey R, et al. Allergen immunotherapy: a practice parameter third update. J Allergy Clin Immunol 2011;127:S1-55.

28. Walker SM, Durham SR, Till SJ, et al. Immunotherapy for allergic rhinitis. Clin Exp Allergy 2011;41:1177-200.

29. Qiu Q, Xu M, Lu C, et al. Safety and efficacy of rush allergen-specific immunotherapy in Chinese allergic rhinitis patients. Int J Immunopathol Pharmacol 2016;29:720-5.

30. Zhou H, Tao QL, Wei JM, Xu G, Cheng L. Trends in Specific Immunotherapy for Allergic Rhinitis: A Survey of Chinese ENT Specialists. Allergy Asthma Immunol Res 2014;6:296-303.

31. Group TCARCR. Chinese Consensus on Allergen Specific Subcutaneous Immunotherapy in Allergic Rhinitis (2015). Chin Arch Otolaryngol Head Neck Surg 2015:379-404.

32. Cardona V, Luengo O, Labrador-Horrillo $M$. Immunotherapy in allergic rhinitis and lower airway outcomes. Allergy 2017;72:35-42.

33. Burks AW, Calderon MA, Casale T, et al. Update on allergy immunotherapy: American Academy of Allergy, Asthma \& Immunology/European Academy of Allergy and Clinical Immunology/PRACTALL consensus report.
J Allergy Clin Immunol 2013;131:1288-96.e3.

34. Calderon MA, Demoly P, Gerth van Wijk R, et al. EAACI: A European Declaration on Immunotherapy. Designing the future of allergen specific immunotherapy. Clin Transl Allergy 2012;2:20.

35. Hankin CS, Cox L. Allergy immunotherapy: what is the evidence for cost saving? Curr Opin Allergy Clin Immunol 2014;14:363-70.

36. Bousquet J, Van Cauwenberge P, Khaltaev N, et al. Allergic rhinitis and its impact on asthma. J Allergy Clin Immunol 2001;108:S147-334.

37. Peng H, Li CW, Lin ZB, et al. Long-term efficacy of specific immunotherapy on house dust mite-induced allergic rhinitis in China. Otolaryngol Head Neck Surg 2013;149:40-6.

38. Des Roches A, Paradis L, Menardo JL, et al. Immunotherapy with a standardized Dermatophagoides pteronyssinus extract. VI. Specific immunotherapy prevents the onset of new sensitizations in children. J Allergy Clin Immunol 1997;99:450-3.

39. Purello-D'Ambrosio F, Gangemi S, Merendino RA, et al. Prevention of new sensitizations in monosensitized subjects submitted to specific immunotherapy or not. A retrospective study. Clin Exp Allergy 2001;31:1295-302.

40. Zolkipli Z, Roberts G, Cornelius V, et al. Randomized controlled trial of primary prevention of atopy using house dust mite allergen oral immunotherapy in early childhood. J Allergy Clin Immunol 2015;136:1541-7.e11.

41. Kristiansen M, Dhami S, Netuveli G, et al. Allergen immunotherapy for the prevention of allergy: A systematic review and meta-analysis. Pediatr Allergy Immunol 2017;28:18-29.

42. Long JZ, Ai R, Xie J, et al. Long-term efficacy of standardized specific subcutaneous immunotherapy on pediatric persistent allergic rhinitis. Chin Arch Otolaryngol Head Neck Surg 2016;23:283-6.

43. Chen Z, Qian Y, Liu S, et al. Onset time and efficacy of sublingual immunotherapy with Dermatophagoides farinae drops in children with allergic rhinitis. Zhonghua Er Bi Yan Hou Tou Jing Wai Ke Za Zhi 2015;50:622-6.

44. Cui L, Wang ZN, Xu ZQ, et al. Efficacy and safety of sublingual immunotherapy of allergic rhinitis in children between 4 and 5 years age group and 11 and 12 years age group. Zhonghua Er Bi Yan Hou Tou Jing Wai Ke Za Zhi 2013;48:17-21.

45. Chen S, Zeng X, Wang L, et al. Effects of house dust mite sublingual immunotherapy in children with allergic rhinitis and asthma. Zhonghua Er Bi Yan Hou Tou Jing Wai Ke 
Za Zhi 2015;50:627-31.

46. Zhao D, Lai X, Tian M, et al. The Functional IgEBlocking Factor Induced by Allergen-Specific Immunotherapy Correlates with IgG4 Antibodies and a Decrease of Symptoms in House Dust Mite-Allergic Children. Int Arch Allergy Immunol 2016;169:113-20.

47. Li X, Wang X, Lin X, et al. Semi-depot house-dust mite allergen extract for Chinese with allergic rhinitis and asthma. Am J Rhinol Allergy 2016;30:201-8.

48. Lin Z, Zhou L, Luo X, et al. Suppression of TIM-1 predicates clinical efficacy of sublingual immunotherapy for allergic rhinitis in children. Int J Pediatr Otorhinolaryngol 2013;77:1345-9.

49. Weng Y, Dongsheng GU, Zeng D, et al. Analysis of the compliance and effectiveness of the allergen specific immunotherapy in patients with allergic rhinitis. J Clin Otorhinolaryngol Head Neck Surg 2016:7:542-5.

50. Song W, Lin X, Xie H, et al. Evaluation of the efficacy and safety of standardized dust mite allergen specific immunotherapy to children with allergic asthma. Lin Chung Er Bi Yan Hou Tou Jing Wai Ke Za Zhi 2013;27:1193-6.

51. Song W, Lin X, Chai R. Efficacy evaluation of standardized dust mite allergen specific immunotherapy to patients of allergic rhinitis. Lin Chung Er Bi Yan Hou Tou Jing Wai Ke Za Zhi 2014;28:300-2.

52. Song W, Lin X, Chai R. Evaluation of long-term effect for house dust mite subcutaneous immunotherapy for patients with allergic rhinitis. Zhonghua Er Bi Yan Hou Tou Jing Wai Ke Za Zhi 2015;50:632-5.

53. Marogna M, Tomassetti D, Bernasconi A, et al. Preventive effects of sublingual immunotherapy in childhood: an open randomized controlled study. Ann Allergy Asthma Immunol 2008;101:206-11.

54. Shao J, Cui YX, Zheng YF, et al. Efficacy and safety of sublingual immunotherapy in children aged 3-13 years with allergic rhinitis. Am J Rhinol Allergy 2014;28:131-9.

55. Wang H, Lin X, Hao C, et al. A double-blind, placebocontrolled study of house dust mite immunotherapy in Chinese asthmatic patients. Allergy 2006;61:191-7.

56. Reha CM, Ebru A. Specific immunotherapy is effective in the prevention of new sensitivities. Allergologia Et Immunopathologia 2007;35:44-51.

57. Inal A, Altintas DU, Yilmaz M, et al. Prevention of new sensitizations by specific immunotherapy in children with rhinitis and/or asthma monosensitized to house dust mite. J Investig Allergol Clin Immunol 2007;17:85-91.

58. Silvestri M, Oddera S, Rossi GA, et al. Sensitization to airborne allergens in children with respiratory symptoms. Ann Allergy Asthma Immunol 1996;76:239-44.

59. Silvestri M, Rossi GA, Cozzani S, et al. Age-dependent tendency to become sensitized to other classes of aeroallergens in atopic asthmatic children. Ann Allergy Asthma Immunol 1999;83:335-40.

60. Drummond M, Brown R, Fendrick AM, et al. Use of pharmacoeconomics information--report of the ISPOR Task Force on use of pharmacoeconomic/health economic information in health-care decision making. Value Health 2003;6:407-16.

61. Ariano R, Berto P, Incorvaia C, et al. Economic evaluation of sublingual immunotherapy vs. symptomatic treatment in allergic asthma. Ann Allergy Asthma Immunol 2009;103:254-9.

62. Ariano R, Berto P, Tracci D, et al. Pharmacoeconomics of allergen immunotherapy compared with symptomatic drug treatment in patients with allergic rhinitis and asthma. Allergy Asthma Proc 2006;27:159-63.

63. Berto P, Bassi M, Incorvaia C, et al. Cost effectiveness of sublingual immunotherapy in children with allergic rhinitis and asthma. Eur Ann Allergy Clin Immunol 2005;37:303-8.

64. Berto P, Passalacqua G, Crimi N, et al. Economic evaluation of sublingual immunotherapy vs symptomatic treatment in adults with pollen-induced respiratory allergy: the Sublingual Immunotherapy Pollen Allergy Italy (SPAI) study. Ann Allergy Asthma Immunol 2006;97:615-21.

65. Brüggenjürgen B, Reinhold T, Brehler R, et al. Costeffectiveness of specific subcutaneous immunotherapy in patients with allergic rhinitis and allergic asthma. Ann Allergy Asthma Immunol 2008;101: 316-24.

66. Nasser S, Vestenbaek U, Beriot-Mathiot A, et al. Costeffectiveness of specific immunotherapy with Grazax in allergic rhinitis co-existing with asthma. Allergy 2008;63: 1624-9.

67. Omnes LF, Bousquet J, Scheinmann P, et al. Pharmacoeconomic assessment of specific immunotherapy versus current symptomatic treatment for allergic rhinitis and asthma in France. Eur Ann Allergy Clin Immunol 2007;39:148-56.

68. Bachert C, Noergaard Andreasen J. Cost-Effectiveness of Immunotherapy in the Treatment of Seasonal Allergic Rhinitis: Identifying Product-Specific Parameters of Relevance for Health Care Decision-Makers and Clinicians. Int Arch Allergy Immunol 2015;168:213-7.

69. Cox L. Allergy immunotherapy in reducing healthcare cost. Curr Opin Otolaryngol Head Neck Surg 
2015;23:247-54.

70. Hankin CS, Cox L, Bronstone A, et al. Allergy immunotherapy: reduced health care costs in adults and children with allergic rhinitis. J Allergy Clin Immunol 2013;131:1084-91.

71. Simoens S. The cost-effectiveness of immunotherapy for respiratory allergy: a review. Allergy 2012;67:1087-105.

72. Chen J, Xiang J, Wang Y, et al. Health economics analysis of specific immunotherapy in allergic rhinitis accompanied with asthma). Lin Chung Er Bi Yan Hou Tou Jing Wai Ke Za Zhi 2013;27:925-8.

73. Zuberbier T, Bachert C, Bousquet PJ, et al. GA2LEN/ EAACI pocket guide for allergen-specific immunotherapy for allergic rhinitis and asthma. Allergy 2010;65:1525-30.

74. Alvarez-Cuesta E, Bousquet J, Canonica GW, et al. Standards for practical allergen-specific immunotherapy. Allergy 2006;61 Suppl 82:1-20.

75. Passalacqua G, Canonica GW. Allergen Immunotherapy: History and Future Developments. Immunol Allergy Clin North Am 2016;36:1-12.

76. Scadding GK, Brostoff J. Low dose sublingual therapy in patients with allergic rhinitis due to house dust mite. Clin Allergy 1986;16:483-91.

77. Jutel M, Bartkowiak-Emeryk M, Breborowicz A, et al. Sublingual immunotherapy (SLIT)--indications, mechanism, and efficacy: Position paper prepared by the Section of Immunotherapy, Polish Society of Allergy. Ann Agric Environ Med 2016;23:44-53.

78. Senti G, Prinz Vavricka BM, Erdmann I, et al. Intralymphatic allergen administration renders specific immunotherapy faster and safer: a randomized controlled trial. Proc Natl Acad Sci U S A 2008;105:17908-12.

79. Sindher S, Fleischer DM, Spergel JM. Advances in the Treatment of Food Allergy: Sublingual and Epicutaneous Immunotherapy. Immunol Allergy Clin North Am 2016;36:39-54.

80. Feuille E, Nowak-Wegrzyn A. Oral Immunotherapy for Food Allergies. Ann Nutr Metab 2016;68 Suppl 1:19-31.

81. Nickelsen JA, Goldstein S, Mueller U, et al. Local intranasal immunotherapy for ragweed allergic rhinitis. I. Clinical response. J Allergy Clin Immunol 1981;68:33-40.

82. Canonica GW, Passalacqua G. Noninjection routes for immunotherapy. J Allergy Clin Immunol 2003;111:43748; quiz 49.

83. Arshad SH. An update on allergen immunotherapy. Clin Med (Lond) 2016;16:584-7.

84. Xu LZ, Yang LT, Qiu SQ, et al. Combination of specific allergen and probiotics induces specific regulatory B cells and enhances specific immunotherapy effect on allergic rhinitis. Oncotarget 2016;7:54360-9.

85. Luo X, Hong $\mathrm{H}$, Tang J, et al. Increased Expression of miR-146a in Children With Allergic Rhinitis After Allergen-Specific Immunotherapy. Allergy Asthma Immunol Res 2016;8:132-40.

86. Akdis CA, Akdis M. Mechanisms of allergen-specific immunotherapy. J Allergy Clin Immunol 2011;127:1827; quiz 8-9.

87. Tarzi M, Klunker S, Texier C, et al. Induction of interleukin-10 and suppressor of cytokine signalling-3 gene expression following peptide immunotherapy. Clin Exp Allergy 2006;36:465-74.

88. Alexander C, Ying S, B Kay A, et al. Fel d 1-derived $T$ cell peptide therapy induces recruitment of CD4+ CD25+;CD4+ interferon-gamma+ $T$ helper type 1 cells to sites of allergen-induced late-phase skin reactions in catallergic subjects. Clin Exp Allergy 2005;35:52-8.

89. Woo HY, Kim YS, Kang NI, et al. Mechanism for acute oral desensitization to antibiotics. Allergy 2006;61:954-8.

90. Bussmann C, Xia J, Allam JP, et al. Early markers for protective mechanisms during rush venom immunotherapy. Allergy 2010;65:1558-65.

91. Larché M, Akdis CA, Valenta R. Immunological mechanisms of allergen-specific immunotherapy. Nat Rev Immunol 2006;6:761-71.

92. Lambrecht BN, Pauwels RA, Fazekas De St Groth B. Induction of rapid $\mathrm{T}$ cell activation, division, and recirculation by intratracheal injection of dendritic cells in a TCR transgenic model. J Immunol 2000;164:2937-46.

93. Jonuleit H, Schmitt E, Schuler G, et al. Induction of interleukin 10-producing, nonproliferating CD4(+) T cells with regulatory properties by repetitive stimulation with allogeneic immature human dendritic cells. J Exp Med 2000;192:1213-22.

94. de Heer HJ, Hammad H, Soullie T, et al. Essential role of lung plasmacytoid dendritic cells in preventing asthmatic reactions to harmless inhaled antigen. J Exp Med 2004;200:89-98.

95. Akdis CA, Blesken T, Akdis M, et al. Role of interleukin 10 in specific immunotherapy. J Clin Invest 1998;102:98-106.

96. Nouri-Aria KT, Wachholz PA, Francis JN, et al. Grass pollen immunotherapy induces mucosal and peripheral IL-10 responses and blocking IgG activity. J Immunol 2004;172:3252-9.

97. Akdis M, Verhagen J, Taylor A, et al. Immune responses in healthy and allergic individuals are characterized by a fine balance between allergen-specific $T$ regulatory 1 and $T$ 
helper 2 cells. J Exp Med 2004;199:1567-75.

98. Ling EM, Smith T, Nguyen XD, et al. Relation of CD4+CD25+ regulatory T-cell suppression of allergendriven T-cell activation to atopic status and expression of allergic disease. Lancet 2004;363:608-15.

99. Jutel M, Akdis M, Budak F, et al. IL-10 and TGF-beta cooperate in the regulatory $T$ cell response to mucosal allergens in normal immunity and specific immunotherapy. Eur J Immunol 2003;33:1205-14.

100. Verhoef A, Alexander C, Kay AB, et al. T cell epitope immunotherapy induces a CD4+ $\mathrm{T}$ cell population with regulatory activity. PLoS Med 2005;2:e78.

101. Varney VA, Hamid QA, Gaga M, et al. Influence of grass pollen immunotherapy on cellular infiltration and cytokine mRNA expression during allergen-induced late-phase cutaneous responses. J Clin Invest 1993;92:644-51.

102. Ebner C, Siemann U, Bohle B, et al. Immunological changes during specific immunotherapy of grass pollen allergy: reduced lymphoproliferative responses to allergen and shift from TH2 to TH1 in T-cell clones specific for Phl p 1, a major grass pollen allergen. Clin Exp Allergy 1997;27:1007-15.

103.Akdis CA, Akdis M, Blesken T, et al. Epitope-specific $\mathrm{T}$ cell tolerance to phospholipase $\mathrm{A} 2$ in bee venom immunotherapy and recovery by IL-2 and IL-15 in vitro. J Clin Invest 1996;98:1676-83.

104. Akdis CA, Blaser K. IL-10-induced anergy in peripheral $T$ cell and reactivation by microenvironmental cytokines: two key steps in specific immunotherapy. FASEB J 1999;13:603-9.

105. Francis JN, Till SJ, Durham SR. Induction of IL10+CD4+CD25+ T cells by grass pollen immunotherapy. $\mathrm{J}$ Allergy Clin Immunol 2003;111:1255-61.

106. Bellinghausen I, Metz G, Enk AH, et al. Insect venom immunotherapy induces interleukin-10 production and a Th2-to-Th1 shift, and changes surface marker expression in venom-allergic subjects. Eur J Immunol 1997;27:1131-9.

107.Pereira EA, Silva DA, Cunha-Junior JP, et al. IgE, IgG1, and IgG4 antibody responses to Blomia tropicalis in atopic patients. Allergy 2005;60:401-6.

108. Lichtenstein LM, Holtzman NA, Burnett LS. A quantitative in vitro study of the chromatographic distribution and immunoglobulin characteristics of human blocking antibody. J Immunol 1968;101:317-24.

109. Cooke RA, Barnard JH, Hebald S, et al. Serological Evidence of Immunity with Coexisting Sensitization in a Type of Human Allergy (Hay Fever). J Exp Med $1935 ; 62: 733-50$.
110. Golden DB, Meyers DA, Kagey-Sobotka A, et al. Clinical relevance of the venom-specific immunoglobulin $\mathrm{G}$ antibody level during immunotherapy. J Allergy Clin Immunol 1982;69:489-93.

111. Müller U, Helbling A, Bischof M. Predictive value of venom-specific IgE, IgG and IgG subclass antibodies in patients on immunotherapy with honey bee venom. Allergy 1989;44:412-8.

112. Djurup R, Malling HJ. High IgG4 antibody level is associated with failure of immunotherapy with inhalant allergens. Clin Allergy 1987;17:459-68.

113. Gafvelin G, Thunberg S, Kronqvist M, et al. Cytokine and antibody responses in birch-pollen-allergic patients treated with genetically modified derivatives of the major birch pollen allergen Bet v 1. Int Arch Allergy Immunol 2005;138:59-66.

114. Hirahara K, Tatsuta T, Takatori T, et al. Preclinical evaluation of an immunotherapeutic peptide comprising 7 T-cell determinants of Cry j 1 and Cry j 2, the major Japanese cedar pollen allergens. J Allergy Clin Immunol 2001;108:94-100.

115. King TP, Jim SY, Monsalve RI, et al. Recombinant allergens with reduced allergenicity but retaining immunogenicity of the natural allergens: hybrids of yellow jacket and paper wasp venom allergen antigen $5 \mathrm{~s}$. J Immunol 2001;166:6057-65.

116. Soyer OU, Akdis M, Akdis CA. Mechanisms of subcutaneous allergen immunotherapy. Immunol Allergy Clin North Am 2011;31:175-90, vii-viii.

117. Bush RK. Advances in allergen immunotherapy in 2015. J Allergy Clin Immunol 2016;138:1284-91.

118. Canonica GW, Cox L, Pawankar R, et al. Sublingual immunotherapy: World Allergy Organization position paper 2013 update. World Allergy Organ J 2014;7:6.

119. Comité Nacional de Alergia de la Sociedad Argentina de Pediatría, Saranz RJ, Lozano A, et al. Allergen immunotherapy for prevention and treatment of respiratory allergy in childhood. Arch Argent Pediatr 2010;108:258-65.

120. Bozek A, Jarzab J, Bednarski P. The effect of allergenspecific immunotherapy on offspring. Allergy Asthma Proc 2016;37:59-63.

121. Oykhman P, Kim HL, Ellis AK. Allergen immunotherapy in pregnancy. Allergy Asthma Clin Immunol 2015;11:31.

122. Van Hoecke H, Van Cauwenberge P, Thas O, et al. The ARIA guidelines in specialist practice: a nationwide survey. Rhinology 2010;48:28-34.

123. Madsen F. EAACI 'Standards for practical allergen-specific 
immunotherapy'. Allergy 2007;62:332.

124.Jutel M, Agache I, Bonini S, et al. International consensus on allergy immunotherapy. J Allergy Clin Immunol 2015;136:556-68.

125. Subspecialty Group of Rhinology, Editorial Board of Chinese Journal of Otorhinolaryngology Head and Neck Surgery;Subspecialty Group of Rhinology, et al. Expert consensus on allergen specific immunotherapy of allergic rhinitis. Zhonghua Er Bi Yan Hou Tou Jing Wai Ke Za Zhi 2011;46:976-80.

126. Subspecialty Group of Rhinology, Editorial Board of Chinese Journal of Otorhinolaryngology Head and Neck Surgery;Subspecialty Group of Rhinology, et al. Chinese guidelines for diagnosis and treatment of allergic rhinitis. Zhonghua Er Bi Yan Hou Tou Jing Wai Ke Za Zhi 2016;51:6-24.

127. Viswanathan RK, Busse WW. Allergen immunotherapy in allergic respiratory diseases: from mechanisms to metaanalyses. Chest 2012;141:1303-14.

128. Feng B, Wu J, Chen B, et al. Efficacy and safety of sublingual immunotherapy for allergic rhinitis in pediatric patients: A meta-analysis of randomized controlled trials. Am J Rhinol Allergy 2017;31:27-35.

129. Okubo K, Kurono Y, Ichimura K, et al. Japanese guidelines for allergic rhinitis 2017. Allergol Int 2017;66:205-19.

130.Zhang L, Wang X, Wang C. The assessment and management of anaphylaxis. Zhonghua Er Bi Yan Hou Tou Jing Wai Ke Za Zhi 2015;50:619-21.

131.Duan C, Tian Y, Zhang F, et al. Efficacy of sublingual immunotherapy and subcutaneous immunotherapy in children with allergic rhinitis. J Clin Otorhinolaryngol Head Neck Surg 2016;17:1354-8.

132. Dai L, Huang Y, Wang Y, et al. Serious systemic adverse events associated with allergen-specific immunotherapy in children with asthma. Zhongguo Dang Dai Er Ke Za Zhi 2014;16:58-61.

133. Cui L, Xia Z, Wang Z, et al. Efficacy of sublingual immunotherapy with Dermatophagoides farina drops in monosensitized and polysensitized patients with allergic rhinitis. Lin Chung Er Bi Yan Hou Tou Jing Wai Ke Za Zhi 2015;29:1094-7.

134.Li P, Li Q, Huang Z, et al. Efficacy and safety of house dust mite sublingual immunotherapy in monosensitized and polysensitized children with respiratory allergic diseases. Int Forum Allergy Rhinol 2014;4:796-801.

135. Yukselen A. Allergen-specific immunotherapy in pediatric allergic asthma. Asia Pac Allergy 2016;6:139-48.

136. Metzger WJ, Turner E, Patterson R. The safety of immunotherapy during pregnancy. J Allergy Clin Immunol 1978;61:268-72.

137. Shaikh WA. A retrospective study on the safety of immunotherapy in pregnancy. Clin Exp Allergy 1993;23:857-60.

138. Shaikh WA, Shaikh SW. A prospective study on the safety of sublingual immunotherapy in pregnancy. Allergy 2012;67:741-3.

139.Pitsios C, Demoly P, Bilo MB, et al. Clinical contraindications to allergen immunotherapy: an EAACI position paper. Allergy 2015;70:897-909.

140.Jutel M, Agache I, Bonini S, et al. International Consensus on Allergen Immunotherapy II: Mechanisms, standardization, and pharmacoeconomics. J Allergy Clin Immunol 2016;137:358-68.

141.King TP, Hoffman D, Lowenstein H, et al. Allergen nomenclature. Allergy 1995;50:765-74.

142. Spangfort MD, Larsen JN. Standardization of allergenspecific immunotherapy vaccines. Immunol Allergy Clin North Am 2006;26:191-206, v-vi.

143. Løwenstein H. Selection of reference preparation. IUIS reference preparation criteria. International Union of Immunological Societies. Arb Paul Ehrlich Inst Georg Speyer Haus Ferdinand Blum Inst Frankf A M 1987;(80):75-8.

144.Zheng YW, Lai XX, Zhao DY, et al. Indoor Allergen Levels and Household Distributions in Nine Cities Across China. Biomed Environ Sci 2015;28:709-17.

145. Nahler G. Nordic Council on Medicines (NLN). In: Dictionary of Pharmaceutical Medicine. Vienna: Springer, 2009.

146. Baer H, Godfrey H, Maloney CJ, et al. The potency and antigen $\mathrm{E}$ content of commercially prepared ragweed extracts. J Allergy 1970;45:347-54.

147.Baer H, Maloney CJ, Norman PS, et al. The potency and Group I antigen content of six commercially prepared grass pollen extracts. J Allergy Clin Immunol 1974;54:157-64.

148.Zheng YW, Li J, Lai XX, et al. Allergen micro-array detection of specific IgE-reactivity in Chinese allergy patients. Chin Med J (Engl) 2011;124:4350-4.

149.Zhao ZH, Elfman L, Wang ZH, et al. A comparative study of asthma, pollen, cat and dog allergy among pupils and allergen levels in schools in Taiyuan city, China, and Uppsala, Sweden. Indoor Air 2006;16:404-13.

150.Hao GD, Zheng YW, Gjesing B, et al. Prevalence of sensitization to weed pollens of Humulus scandens, Artemisia vulgaris, and Ambrosia artemisiifolia in northern 
China. J Zhejiang Univ Sci B 2013;14:240-6.

151. Tang R, Sun JL, Yin J, et al. Artemisia allergy research in China. Biomed Res Int 2015;2015:179426.

152. Leng X, Ye ST. One year observation of immunotherapy for Artemisia hay fever in China: a clinical and immunological study. Asian Pac J Allergy Immunol 1987;5:167-72.

153.Lu D, Zhau P, Yu Q, et al. Airway provocation test with ragweed pollen extract in Chinese asthmatics. Asian Pac J Allergy Immunol 1994;12:125-9.

154. Chen Z, Yang Y, Chen X, et al. Characterization of two pollen allergens of the London plane tree in Shanghai. Iran J Allergy Asthma Immunol 2015;14:139-48.

155.Hao GD, Zheng YW, Wang ZX, et al. High correlation of specific $\mathrm{IgE}$ sensitization between birch pollen, soy and apple allergens indicates pollen-food allergy syndrome among birch pollen allergic patients in northern China. J Zhejiang Univ Sci B 2016;17:399-404.

156.Jiang N, Yin J, Mak P, et al. Occupational Allergy to Peach (Prunus persica) Tree Pollen and Potential CrossReactivity between Rosaceae Family Pollens. Iran J Allergy Asthma Immunol 2015;14:483-92.

157.Luo W, Huang H, Zheng $\mathrm{P}$, et al. Major grass pollen allergens and components detected in a southern Chinese cohort of patients with allergic rhinitis and/or asthma. Mol Immunol 2016;78:105-12.

158.Larsen JN, Dreborg S. Standardization of allergen extracts. Methods Mol Med 2008;138:133-45.

159. Hansen GN, Larsen JN. Immunoelectrophoresis for the characterization of allergen extracts. Methods Mol Med 2008;138:147-65.

160.Henmar H, Frisenette SM, Grosch K, et al. Fractionation of Source Materials Leads to a High Reproducibility of the SQ House Dust Mite SLIT-Tablets. Int Arch Allergy Immunol 2016;169:23-32.

161. Reuter A, Luttkopf D, Vieths S. New frontiers in allergen standardization. Clin Exp Allergy 2009;39:307-9.

162. Scheiner O, Kraft D. Basic and practical aspects of recombinant allergens. Allergy 1995;50:384-91.

163.Cui Y, Jiang Y, Ji Y, et al. Cloning, expression, and analysis of a cDNA coding for the Dermatophagoides farinae group 21 (Der f 21) allergen. Am J Transl Res 2014;6:786-92.

164. Cui Y, Zhou Y, Ma G, et al. Cloning, bioinformatics analysis, and expression of the dust mite allergen Der f 5 of Dermatophagoides farinae. Braz J Med Biol Res 2012;45:746-52.

165. Cui Y, Zhou Y, Shi W, et al. Molecular cloning, expression, sequence analyses of dust mite allergen Der $\mathrm{f} 6$ and its
IgE-binding reactivity with mite allergic asthma patients in southeast China. Mol Biol Rep 2012;39:961-8.

166. Cui Y, Zhou Y, Wang Y, et al. The group 10 allergen of Dermatophagoides farinae (Acari: Pyroglyphidae): cDNA cloning, sequence analysis, and expression in Escherichia coli BL21. J Med Entomol 2013;50:205-8.

167. Cui YB, Cai HX, Li L, et al. Cloning, sequence analysis and expression in E. coli of the group 3 allergen of Dermatophagoides farinae. Chin Med J (Engl) 2009;122:2657-61.

168. Cui YB, Cai HX, Zhou Y, et al. Cloning, expression, and characterization of Der $\mathrm{f} 7$, an allergen of Dermatophagoides farinae from China. J Med Entomol 2010;47:868-76.

169. Cui YB, Cai HX, Zhou Y, et al. The Dermatophagoides farinae group 22 allergen: cloning and expression in Escherichia coli. Int Forum Allergy Rhinol 2015;5:794-800.

170. Cui YB, Zhou Y, Wang N, et al. Expression, cloning, and $\mathrm{IgE}$-binding of the full-length dust mite allergen Der $\mathrm{f} 8$. Immunol Res 2014;60:60-8.

171.Yu-bao C, Zhou Y, Weihong S, et al. Cloning, expression, and analysis of the group 2 allergen from Dermatophagoides farinae from China. An Acad Bras Cienc 2010;82:941-51.

172. Bernstein IL, Li JT, Bernstein DI, et al. Allergy diagnostic testing: an updated practice parameter. Ann Allergy Asthma Immunol 2008;100:S1-148.

173. Sekerel BE, Sahiner UM, Bousquet J, et al. Practical guide to skin prick tests in allergy to aeroallergens: some concerns. Allergy 2012;67:442; author reply 443.

174.Li QM, Peng XY, Li XP. Analysis of skin prickle test with standardized antigen and its relationship with degree of symptoms in patients with allergic rhinitis. Chin J Otorhinolaryngol Skull Base Surg 2010;16:121-3.

175. Canonica GW, Bousquet J, Casale T, et al. Sub-lingual immunotherapy: world allergy organization position paper 2009. World Allergy Organ J 2009;2:233-81.

176. Ahlstedt S, Murray CS. In vitro diagnosis of allergy: how to interpret $\mathrm{IgE}$ antibody results in clinical practice. Prim Care Respir J 2006;15:228-36.

177.Xu YY, Liu HY, Cheng KJ, et al. Relativity of the intensity of specific $\mathrm{IgE}$ and skin prick test in patients with perennial allergic rhinitis. Chin Arch Otolaryngol Head Neck Surg 2010;17:628-30.

178. Zhang Y, Liu CY, Zhang L. Correlation Analysis of Serum Specific IgE and Skin Prick Test in Allergic Rhinitis Patients. J Cap Med Univ 2009;30:733-6.

179. Tabar AI, Echechipia S, Garcia BE, et al. Doubleblind comparative study of cluster and conventional 
immunotherapy schedules with Dermatophagoides pteronyssinus. J Allergy Clin Immunol 2005;116:109-18.

180. Rieker-Schwienbacher J, Nell MJ, Diamant Z, et al. Openlabel parallel dose tolerability study of three subcutaneous immunotherapy regimens in house dust mite allergic patients. Clin Transl Allergy 2013;3:16.

181. Temiño VM, Wu P, Konig J, et al. Safety of multiple aeroallergen rush immunotherapy using a modified schedule. Allergy Asthma Proc 2013;34:255-60.

182. Calderón MA, Cox L, Casale TB, et al. Multipleallergen and single-allergen immunotherapy strategies in polysensitized patients: looking at the published evidence. J Allergy Clin Immunol 2012;129:929-34.

183. Bauer CS, Rank MA. Comparative efficacy and safety of subcutaneous versus sublingual immunotherapy. J Allergy Clin Immunol 2014;134:765.e2.

184. Casale TB, Stokes JR. Immunotherapy: what lies beyond. J Allergy Clin Immunol 2014;133:612-9: quiz 620.

185.von Moos S, Johansen P, Tay F, et al. Comparing safety of abrasion and tape-stripping as skin preparation in allergenspecific epicutaneous immunotherapy. J Allergy Clin Immunol 2014;134:965-7.e4.

186. Roche AM, Wise SK. Subcutaneous immunotherapy. Int Forum Allergy Rhinol 2014;4 Suppl 2:S51-4.

187. Marogna M, Spadolini I, Massolo A, et al. Long-lasting effects of sublingual immunotherapy according to its duration: a 15-year prospective study. J Allergy Clin Immunol 2010;126:969-75.

188. Durham SR, Walker SM, Varga EM, et al. Long-term clinical efficacy of grass-pollen immunotherapy. N Engl J Med 1999;341:468-75.

189. Des Roches A, Paradis L, Knani J, et al. Immunotherapy with a standardized Dermatophagoides pteronyssinus extract. V. Duration of the efficacy of immunotherapy after its cessation. Allergy 1996;51:430-3.

190.de Groot H, Bijl A. Anaphylactic reaction after the first dose of sublingual immunotherapy with grass pollen tablet. Allergy 2009;64:963-4.

191. Cox LS, Larenas Linnemann D, Nolte H, et al. Sublingual immunotherapy: a comprehensive review. J Allergy Clin Immunol 2006;117:1021-35.

192.Lombardi C, Gargioni S, Cottini M, et al. The safety of sublingual immunotherapy with one or more allergens in adults. Allergy 2008;63:375-6.

193.Passalacqua G, Pawankar R, Baena-Cagnani CE, et al. Sublingual immunotherapy: where do we stand? Present and future. Curr Opin Allergy Clin Immunol 2009;9:1-3. 194.Feng S, Xu Y, Ma R, et al. Cluster subcutaneous allergen specific immunotherapy for the treatment of allergic rhinitis: a systematic review and meta-analysis. PLoS One 2014;9:e86529.

195. Nieto García A, Nevot Falco S, Carrillo Diaz T, et al. Safety of cluster specific immunotherapy with a modified high-dose house dust mite extract. Eur Ann Allergy Clin Immunol 2013;45:78-83.

196. Klimek L, Uhlig J, Mosges R, et al. A high polymerized grass pollen extract is efficacious and safe in a randomized double-blind, placebo-controlled study using a novel updosing cluster-protocol. Allergy 2014;69:1629-38.

197. Serrano P, Algorta J, Martinez A, et al. Prospective safety study of immunotherapy administered in a cluster schedule. J Investig Allergol Clin Immunol 2004;14:312-9.

198. Pfaar O, Klimek L, Fischer I, et al. Safety of two cluster schedules for subcutaneous immunotherapy in allergic rhinitis or asthma patients sensitized to inhalant allergens. Int Arch Allergy Immunol 2009;150:102-8.

199. Subiza J, Feliu A, Subiza JL, et al. Cluster immunotherapy with a glutaraldehyde-modified mixture of grasses results in an improvement in specific nasal provocation tests in less than 2.5 months of treatment. Clin Exp Allergy 2008;38:987-94.

200. Bukantz SC, Bagg AS, Lockey RF. Adverse effects and fatalities associated with subcutaneous allergen immunotherapy. Clin Allergy Immunol 2008;21:455-68.

201. Stewart GE 2nd, Lockey RF. Systemic reactions from allergen immunotherapy. J Allergy Clin Immunol 1992;90:567-78.

202. Calderón MA, Vidal C, Rodríguez Del Río P, et al. European Survey on Adverse Systemic Reactions in Allergen Immunotherapy (EASSI): a real-life clinical assessment. Allergy 2017;72:462-72.

203. Cox L. Accelerated immunotherapy schedules: review of efficacy and safety. Ann Allergy Asthma Immunol 2006;97:126-37;quiz 137-40, 202.

204.Roll A, Hofbauer G, Ballmer-Weber BK, et al. Safety of specific immunotherapy using a four-hour ultra-rush induction scheme in bee and wasp allergy. J Investig Allergol Clin Immunol 2006;16:79-85.

205.Harvey SM, Laurie S, Hilton K, et al. Safety of rush immunotherapy to multiple aeroallergens in an adult population. Ann Allergy Asthma Immunol 2004;92:414-9.

206.Pfaar O, Urry Z, Robinson DS, et al. A randomized placebo-controlled trial of rush preseasonal depigmented polymerized grass pollen immunotherapy. Allergy 2012;67:272-9.

207.Nagao M, Hiraguchi Y, Hosoki K, et al. Allergen-induced 
basophil CD203c expression as a biomarker for rush immunotherapy in patients with Japanese cedar pollinosis. Int Arch Allergy Immunol 2008;146 Suppl 1:47-53.

208. Roger A, Justicia JL, Navarro LA, et al. Observational study of the safety of an ultra-rush sublingual immunotherapy regimen to treat rhinitis due to house dust mites. Int Arch Allergy Immunol 2011;154:69-75.

209. Smits WL, Giese JK, Letz KL, et al. Safety of rush immunotherapy using a modified schedule: a cumulative experience of 893 patients receiving multiple aeroallergens. Allergy Asthma Proc 2007;28:305-12.

210.Xie W, Zhang H, Chen J, et al. Clinical efficacy and safety of rush immunotherapy in patients with allergic rhinitis. Zhonghua Er Bi Yan Hou Tou Jing Wai Ke Za Zhi 2015;50:641-5.

211. Brozek JL, Bousquet J, Baena-Cagnani CE, et al. Allergic Rhinitis and its Impact on Asthma (ARIA) guidelines: 2010 revision. J Allergy Clin Immunol 2010;126:466-76.

212.Wang DH, Chen L, Cheng L, et al. Fast onset of action of sublingual immunotherapy in house dust mite-induced allergic rhinitis: a multicenter, randomized, double-blind, placebo-controlled trial. Laryngoscope 2013;123:1334-40.

213. Wang C, Zhang L. Specific immunotherapy for allergic rhinitis in children. Curr Opin Otolaryngol Head Neck Surg 2014;22:487-94.

214. Zhang Y, Liu C, Han D, et al. Correlation of routine examinations for the diagnosis of house dust mite allergic rhinitis. ORL J Otorhinolaryngol Relat Spec 2011;73:182-8.

215. National Cooperative Group on Childhood Asthma; Institute of Environmental Health and Related Product Safety, Chinese Center for Disease Control and Prevention, et al. Third nationwide survey of childhood asthma in urban areas of China. Zhonghua Er Ke Za Zhi 2013;51:729-35.

216. Masuda S, Fujisawa T, Katsumata H, et al. High prevalence and young onset of allergic rhinitis in children with bronchial asthma. Pediatr Allergy Immunol 2008;19:517-22.

217. Hamouda S, Karila C, Connault T, et al. Allergic rhinitis in children with asthma: a questionnaire-based study. Clin Exp Allergy 2008;38:761-6.

218. de Groot EP, Nijkamp A, Duiverman EJ, Brand PL. Allergic rhinitis is associated with poor asthma control in children with asthma. Thorax 2012;67:582-7.

219. Bousquet J, Gaugris S, Kocevar VS, et al. Increased risk of asthma attacks and emergency visits among asthma patients with allergic rhinitis: a subgroup analysis of the investigation of montelukast as a partner agent for complementary therapy. Clin Exp Allergy 2005;35:723-7.
220.Lohia S, Schlosser RJ, Soler ZM. Impact of intranasal corticosteroids on asthma outcomes in allergic rhinitis: a meta-analysis. Allergy 2013;68:569-79.

221. Saporta D. Efficacy of sublingual immunotherapy versus subcutaneous injection immunotherapy in allergic patients. J Environ Public Health 2012;2012:492405.

222. Miraglia Del Giudice M, Marseglia GL, Leonardi S, et al. Fractional exhaled nitric oxide measurements in rhinitis and asthma in children. Int J Immunopathol Pharmacol 2011;24:29-32.

223. Braido F, Baiardini I, Balestracci S, et al. Does asthma control correlate with quality of life related to upper and lower airways? A real life study. Allergy 2009;64:937-43.

224. Chan AH, Stewart AW, Harrison J, et al. The effect of an electronic monitoring device with audiovisual reminder function on adherence to inhaled corticosteroids and school attendance in children with asthma: a randomised controlled trial. Lancet Respir Med 2015;3:210-9.

225. Rhee H, Allen J, Mammen J, et al. Mobile phone-based asthma self-management aid for adolescents (mASMAA): a feasibility study. Patient Prefer Adherence 2014;8:63-72.

226. Ciprandi G, Klersy C, Cirillo I, et al. Quality of life in allergic rhinitis: relationship with clinical, immunological, and functional aspects. Clin Exp Allergy 2007;37:1528-35.

227. Pfaar O, Anders C, Klimek L. Clinical outcome measures of specific immunotherapy. Curr Opin Allergy Clin Immunol 2009;9:208-13.

228. Canonica GW, Baena-Cagnani CE, Bousquet J, et al. Recommendations for standardization of clinical trials with Allergen Specific Immunotherapy for respiratory allergy. A statement of a World Allergy Organization (WAO) taskforce. Allergy 2007;62:317-24.

229. Mosbech H, Canonica GW, Backer V, et al. SQ house dust mite sublingually administered immunotherapy tablet (ALK) improves allergic rhinitis in patients with house dust mite allergic asthma and rhinitis symptoms. Ann Allergy Asthma Immunol 2015;114:134-40.

230. Baiardini I, Braido F, Brandi S, et al. Allergic diseases and their impact on quality of life. Ann Allergy Asthma Immunol 2006;97:419-28; quiz 429-30, 476.

231.Juniper EF, Guyatt GH. Development and testing of a new measure of health status for clinical trials in rhinoconjunctivitis. Clin Exp Allergy 1991;21:77-83.

232. Malling HJ. Criteria for clinical efficacy--readout and monitoring of clinical studies. Arb Paul Ehrlich Inst Bundesamt Sera Impfstoffe Frankf A M 2003;(94):119-23; discussion 123-5.

233. Niu CK, Chen WY, Huang JL, et al. Efficacy of sublingual 
immunotherapy with high-dose mite extracts in asthma: a multi-center, double-blind, randomized, and placebocontrolled study in Taiwan. Respir Med 2006;100:1374-83.

234. Tsai TC, Lu JH, Chen SJ, et al. Clinical efficacy of house dust mite-specific immunotherapy in asthmatic children. Pediatr Neonatol 2010;51:14-8.

235. Tsai YG, Chien JW, Chen WL, et al. Induced apoptosis of TH2 lymphocytes in asthmatic children treated with Dermatophagoides pteronyssinus immunotherapy. Pediatr Allergy Immunol 2005;16:602-8.

236. Wang L, Yin J, Fadel R, et al. House dust mite sublingual immunotherapy is safe and appears to be effective in moderate, persistent asthma. Allergy 2014;69:1181-8.

237. Chen ZG, Li M, Chen YF, et al. Effects of dermatophagoides pteronyssinus allergen-specific immunotherapy on the serum interleukin-13 and pulmonary functions in asthmatic children. Chin Med J (Engl) 2009;122:1157-61.

238. Akdis M, Akdis CA. Mechanisms of allergen-specific immunotherapy: multiple suppressor factors at work in immune tolerance to allergens. J Allergy Clin Immunol 2014;133:621-31.

239. Francis JN. The facilitated antigen binding (FAB) assay--a protocol to measure allergen-specific inhibitory antibody activity. Methods Mol Med 2008;138:255-61.

240. Shamji MH, Ljorring C, Francis JN, et al. Functional rather than immunoreactive levels of IgG4 correlate closely with clinical response to grass pollen immunotherapy. Allergy 2012;67:217-26.

241.James LK, Shamji MH, Walker SM, et al. Long-term tolerance after allergen immunotherapy is accompanied by selective persistence of blocking antibodies. J Allergy Clin Immunol 2011;127:509-16.e1-5.

242. Mastrandrea F, Serio G, Minardi A, et al. IgE responses to Dermatophagoides pteronyssinus native major allergens Der p 1 and Der p 2 during long-term specific immunotherapy. Allergy 1997;52:1115-9.

243. Di Lorenzo G, Mansueto P, Pacor ML, et al. Evaluation of serum s-IgE/total $\mathrm{IgE}$ ratio in predicting clinical response to allergen-specific immunotherapy. J Allergy Clin Immunol 2009;123:1103-10, 1110.e1-4.

244. Aalberse RC, Stapel SO, Schuurman J, et al. Immunoglobulin G4: an odd antibody. Clin Exp Allergy 2009;39:469-77.

245. Devey ME, Wilson DV, Wheeler AW. The IgG subclasses of antibodies to grass pollen allergens produced in hay fever patients during hyposensitization. Clin Allergy 1976;6:227-36.

246. Aalberse R. The role of IgG antibodies in allergy and immunotherapy. Allergy 2011;66 Suppl 95:28-30.

247.Pilette C, Nouri-Aria KT, Jacobson MR, et al. Grass pollen immunotherapy induces an allergen-specific IgA2 antibody response associated with mucosal TGF-beta expression. J Immunol 2007;178:4658-66.

248. Russell MW, Mansa B. Complement-fixing properties of human IgA antibodies. Alternative pathway complement activation by plastic-bound, but not specific antigenbound, IgA. Scand J Immunol 1989;30:175-83.

249. Epstein TG, Liss GM, Murphy-Berendts K, et al. AAAAI/ACAAI surveillance study of subcutaneous immunotherapy, years 2008-2012: an update on fatal and nonfatal systemic allergic reactions. J Allergy Clin Immunol Pract 2014;2:161-7.

250. Kannan JA, Epstein TG. Immunotherapy safety: what have we learned from surveillance surveys? Curr Allergy Asthma Rep 2013;13:381-8.

251. Confino-Cohen R, Goldberg A. Allergen immunotherapyinduced biphasic systemic reactions: incidence, characteristics, and outcome: a prospective study. Ann Allergy Asthma Immunol 2010;104:73-8.

252.Kim L, Nevis I, Potts R, et al. Patients on subcutaneous allergen immunotherapy are at risk of intramuscular injections. Allergy Asthma Clin Immunol 2014;10:22.

253. Lieberman $P$. The risk and management of anaphylaxis in the setting of immunotherapy. Am J Rhinol Allergy 2012;26:469-74.

254. Nelson BL, Dupont LA, Reid MJ. Prospective survey of local and systemic reactions to immunotherapy with pollen extracts. Ann Allergy 1986;56:331-4.

255. Prigal SJ. A ten-year study of repository injections of allergens: local reactions and their management. Ann Allergy 1972;30:529-35.

256. Tankersley MS, Butler KK, Butler WK, et al. Local reactions during allergen immunotherapy do not require dose adjustment. J Allergy Clin Immunol 2000;106:840-3.

257. Dong X, Huang N, Li W, et al. Systemic Reactions to Dust Mite Subcutaneous Immunotherapy: A 3-Year Follow-up Study. Allergy Asthma Immunol Res 2016;8:421-7.

258. Calabria CW, Stolfi A, Tankersley MS. The REPEAT study: recognizing and evaluating periodic local reactions in allergen immunotherapy and associated systemic reactions. Ann Allergy Asthma Immunol 2011;106:49-53.

259. Roy SR, Sigmon JR, Olivier J, et al. Increased frequency of large local reactions among systemic reactors during subcutaneous allergen immunotherapy. Ann Allergy Asthma Immunol 2007;99:82-6.

260. Qiu Q, Luc C, Chen J, et al. Safety of rush allergen- 
specific immunotherapy in allergic rhinitis patients. Lin Chung Er Bi Yan Hou Tou Jing Wai Ke Za Zhi 2014;28:776-9, 784.

261.Zhang L, Han DM. Allergen-specific subcutaneous immunotherapy for allergic rhinitis. Zhonghua Er Bi Yan Hou Tou Jing Wai Ke Za Zhi 2007;42:711-6.

262. Yi H, Liu Y, Ye J, et al. Clinical observation of the adverse effects of standardized dust mite allergen preparation in the treatment of allergic rhinitis. Lin Chung Er Bi Yan Hou Tou Jing Wai Ke Za Zhi 2014;28:1870-2, 1876.

263. Asero R. Detection of risk factors for systemic adverse reactions to SCIT with natural depot allergen extracts: a retrospective study. Eur Ann Allergy Clin Immunol 2015;47:211-7.

264.Bernstein DI, Wanner M, Borish L, et al, Immunotherapy Committee AAoAA, Immunology. Twelve-year survey of fatal reactions to allergen injections and skin testing:1990-2001. J Allergy Clin Immunol 2004;113:1129-36.

265. Amin HS, Liss GM, Bernstein DI. Evaluation of near-fatal reactions to allergen immunotherapy injections. J Allergy Clin Immunol 2006;117:169-75.

266. Lockey RF, Benedict LM, Turkeltaub PC, et al. Fatalities from immunotherapy (IT) and skin testing (ST). J Allergy Clin Immunol 1987;79:660-77.

267. Reid MJ, Lockey RF, Turkeltaub PC, et al. Survey of fatalities from skin testing and immunotherapy 1985-1989. J Allergy Clin Immunol 1993;92:6-15.

268.Epstein TG, Calabria C, Cox LS, et al. Current Evidence on Safety and Practical Considerations for Administration of Sublingual Allergen Immunotherapy (SLIT) in the United States. J Allergy Clin Immunol Pract 2017;5:34-40.e2.

269. Cox L, Larenas-Linnemann D, Lockey RF, et al. Speaking the same language: The World Allergy Organization Subcutaneous Immunotherapy Systemic Reaction Grading System. J Allergy Clin Immunol 2010;125:569-74, 574.e1-7.

270.Lin ZB, Tian-Ying LI, Chen YQ. Local Adverse Reactions in Patients with Allergic Rhinitis to Dust Mite during Subcutaneous Immunotherapy. Chin J Allergy Clin Immunol 2010;3:212-5.

271.Lin Z, Liu Q, Li T, et al. The effects of house dust mite sublingual immunotherapy in patients with allergic rhinitis according to duration. Int Forum Allergy Rhinol 2016;6:82-7.

272. Chen J, Li B, Zhao Y, et al. A prospective multicenter study of systemic reactions in standardized specific immunotherapy for allergic rhinitis in China. Am J Rhinol Allergy 2014;28:e40-4.

273. Kowalski ML, Ansotegui I, Aberer W, et al. Risk and safety requirements for diagnostic and therapeutic procedures in allergology: World Allergy Organization Statement. World Allergy Organ J 2016;9:33.

274. Muraro A, Roberts G, Worm M, et al. Anaphylaxis: guidelines from the European Academy of Allergy and Clinical Immunology. Allergy 2014;69:1026-45.

275. Erekosima N, Suarez-Cuervo C, Ramanathan M, et al. Effectiveness of subcutaneous immunotherapy for allergic rhinoconjunctivitis and asthma: a systematic review. Laryngoscope 2014;124:616-27.

276. Cox L, Calderon M, Pfaar O. Subcutaneous allergen immunotherapy for allergic disease: examining efficacy, safety and cost-effectiveness of current and novel formulations. Immunotherapy 2012;4:601-16.

277.Zhu L, Lu JH, Xie Q, et al. Compliance and safety evaluation of subcutaneous versus sublingual immunotherapy in mite-sensitized patients with allergic rhinitis. Zhonghua Er Bi Yan Hou Tou Jing Wai Ke Za Zhi 2010;45:444-9.

278. Calderón MA, Simons FE, Malling HJ, et al. Sublingual allergen immunotherapy: mode of action and its relationship with the safety profile. Allergy 2012;67:302-11.

279. Passalacqua G, Baena-Cagnani CE, Bousquet J, et al. Grading local side effects of sublingual immunotherapy for respiratory allergy: speaking the same language. J Allergy Clin Immunol 2013;132:93-8.

280. Cheng L. Sublingual specific immunotherapy for allergic rhinitis. Lin Chung Er Bi Yan Hou Tou Jing Wai Ke Za Zhi 2015;29:197-9.

281. Gidaro GB, Marcucci F, Sensi L, et al. The safety of sublingual-swallow immunotherapy: an analysis of published studies. Clin Exp Allergy 2005;35:565-71.

Cite this article as: Bao Y, Chen J, Cheng L, Guo Y, Hong S, Kong W, Lai H, Li H, Li H, Li J, Li T, Lin X, Liu S, Liu Z, Lou H, Meng J, Qiu Q, Shen K, Tang W, Tao Z, Wang C, Wang X, Wei Q, Xiang L, Xie H, Xu Y, Zhang G, Zhang Y, Zheng Y, Zhi Y, Chen D, Hong H, Li Q, Liu L, Meng Y, Wang N, Wang Y, Zhou Y, Zhang L; Chinese Society of Allergy (CSA) and Chinese Allergic Rhinitis Collaborative Research Group (C2AR2G). Chinese Guideline on allergen immunotherapy for allergic rhinitis. J Thorac Dis 2017;9(11):4607-4650. doi: 10.21037/jtd.2017.10.112 